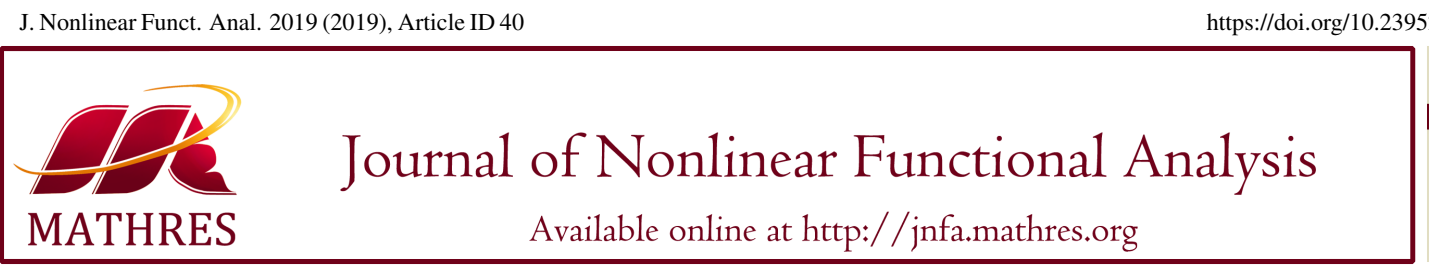

https://doi.org/10.23952/jnfa.2019.40

\title{
MULTI-TERM FRACTIONAL BOUNDARY VALUE PROBLEMS WITH FOUR-POINT BOUNDARY CONDITIONS
}

\author{
BASHIR AHMAD ${ }^{1}$, AHMED ALSAEDI ${ }^{1}$, SOTIRIS K. NTOUYAS ${ }^{2,1, *}$ \\ ${ }^{1}$ Nonlinear Analysis and Applied Mathematics (NAAM)-Research Group, \\ Department of Mathematics, Faculty of Science, King Abdulaziz University, Jeddah 21589, Saudi Arabia \\ ${ }^{2}$ Department of Mathematics, University of Ioannina, 45110 Ioannina, Greece
}

\begin{abstract}
In this paper, we study a new class of boundary value problems for Caputo-type multi-term fractional differential equations and inclusions with four-point boundary conditions. In case of the single-valued problem, we apply Sadovski fixed point theorem, Banach contraction mapping principle and Leray-Schauder nonlinear alternative to derive the existence results, while the multi-valued problem is studied with the aid of nonlinear alternative for contractive maps and Covitz-Nadler fixed point theorem. We illustrate the obtained results with examples. Boundary value problems involving Riemann-Liouville type multi-term fractional differential equations and inclusions are also described.
\end{abstract}

Keywords. Fractional differential equation; Fractional differential inclusion; Boundary value problem; Fixed point theorems.

2010 Mathematics Subject Classification. 34A08, 34A12.

\section{INTRODUCTION}

Fractional differential equations attracted much attention in recent years due to their widespread applications in many fields of science and engineering, such as, fluid flow, signal and image processing, fractals theory, control theory, electromagnetic theory, fitting of experimental data, optics, potential theory, biology, chemistry, diffusion, and viscoelasticity. For a detailed account of applications and recent results on initial and boundary value problems of fractional differential equations and inclusions, we refer the reader to a series of books and papers ([1]-[18]) and the references cited therein.

Equations containing more than one fractional differential term are called multi-term fractional differential equations, which have some concrete applications in many fields. One of the first problems of this kind was considered by Bagley and Torvik to study the motion of a finite sized plate immersed in a

\footnotetext{
${ }^{*}$ Corresponding author.

E-mail addresses: bashirahmad_qau@yahoo.com (B. Ahmad), aalsaedi@hotmail.com (A. Alsaedi), sntouyas@uoi.gr (S.K. Ntouyas).

Received January 14, 2019; Accepted August 28, 2019.
}

(C) 2019 Journal of Nonlinear Functional Analysis 
Newtonian fluid [4]. The general form of the so-called Bagley-Torvik equation is

$$
A y^{\prime \prime}(t)+B^{c} D^{3 / 2} y(t)+C y(t)=f(t),
$$

where $A, B$ and $C$ are constants.

Recently, in $[19,20]$, the authors investigated boundary value problems containing fractional derivatives and integrals of multiple orders in fractional differential equation as well as boundary conditions. Precisely, they considered the following differential equation involving two fractional orders:

$$
\left(\lambda D^{\alpha}+(1-\lambda) D^{\beta}\right) x(t)=f(t, x(t)), \quad t \in(0, T),
$$

supplemented with the boundary conditions:

- two fractional derivatives

$$
x(0)=0, \quad \mu D^{\gamma_{1}} x(T)+(1-\mu) D^{\gamma_{2}} x(T)=\gamma_{3},
$$

or

- two fractional integrals

$$
x(0)=0, \quad \mu I^{\delta_{1}} x(T)+(1-\mu) I^{\delta_{2}} x(T)=\delta_{3},
$$

or

- one fractional derivative and one fractional integral

$$
x(0)=0, \quad \mu D^{\gamma_{1}} x(T)+(1-\mu) I^{\delta_{2}} x(T)=\gamma_{3},
$$

where $D^{\phi}$ is the Riemann-Liouville or Caputo fractional derivative of order $\phi \in\left\{\alpha, \beta, \gamma_{1}, \gamma_{2}\right\}$ such that $1<\alpha, \beta \leq 2$ and $0<\gamma_{1}, \gamma_{2}<\alpha-\beta, \gamma_{3}, \delta_{3} \in \mathbb{R}, I^{\chi}$ is the Riemann-Liouville fractional integral of order $\chi \in\left\{\delta_{1}, \delta_{2}\right\}, 0<\lambda \leq 1,0 \leq \mu \leq 1$ are given constants and $f:[0, T] \times \mathbb{R} \rightarrow \mathbb{R}$ is a continuous function. Existence and uniqueness results were obtained by using classical fixed point theorems.

Also the multi-valued analogue of the above boundary value problems was discussed by considering the differential inclusion:

$$
\left(\lambda D^{\alpha}+(1-\lambda) D^{\beta}\right) x(t) \in F(t, x(t)), \quad t \in(0, T),
$$

equipped with the boundary conditions (1.2)-(1.4), where $F:[0, T] \times \mathbb{R} \rightarrow \mathscr{P}(\mathbb{R})$ is a multivalued function $(\mathscr{P}(\mathbb{R})$ is the family of all nonempty subjects of $\mathbb{R})$.

Here we remark that (1.1) and (1.5) can be called as generalized Bagley-Torvik equation and inclusions respectively with multiple orders.

In a recent work [21], the authors studied existence and stability results for the following three-point boundary value problem with Riemann-Liouville fractional derivatives and integrals

$$
\left\{\begin{array}{l}
\lambda D_{0}^{\alpha} x(t)+D_{0}^{\beta} x(t)=f(t, x(t)), t \in J:=[0, T], \\
x(0)=0, \mu D_{0}^{\gamma_{1}} x(T)+I_{0}^{\gamma_{2}} x(\eta)=\gamma_{3},
\end{array}\right.
$$

where $D_{0}^{\alpha}, D_{0}^{\beta}$ are Riemann-Liouville fractional derivatives with $1<\alpha \leq 2$ and $1 \leq \beta<\alpha, 0 \leq \lambda \leq 1$, $0 \leq \mu \leq 1,0 \leq \gamma_{1} \leq \alpha-\beta, \gamma_{2} \geq 0,0<\eta<T$ and $f:[0, T] \times \mathbb{R} \rightarrow \mathbb{R}$ is a given function. 
In this paper, motivated by the above works, we study a new class of boundary value problems for multi-term fractional differential equations and inclusions supplemented with four-point boundary conditions. Precisely, we investigate the criteria for the existence of solutions for the problems:

$$
\left\{\begin{array}{l}
\lambda{ }^{C} D^{\alpha} x(t)+{ }^{C} D^{\beta} x(t)=f(t, x(t)), t \in J:=[0, T], \\
x^{\prime}(\xi)=v^{C} D^{\gamma} x(\eta), x(T)=\mu I^{\delta} x(\theta), \xi, \eta, \theta \in(0, T),
\end{array}\right.
$$

and

$$
\left\{\begin{array}{l}
\lambda{ }^{C} D^{\alpha} x(t){ }^{C}{ }^{\beta} D^{\beta} x(t) \in F(t, x(t)), t \in J:=[0, T], \\
x^{\prime}(\xi)=v^{C} D^{\gamma} x(\eta), x(T)=\mu I^{\delta} x(\theta), \xi, \eta, \theta \in(0, T),
\end{array}\right.
$$

where ${ }^{C} D^{\chi}$ is Caputo fractional derivatives of order $\chi \in\{\alpha, \beta, \gamma\}, \lambda, v, \mu \in \mathbb{R}, 1<\alpha \leq 2,1<\beta<\alpha$, $0 \leq \gamma<\alpha-\beta<1, \delta>0, I^{\delta}$ is the Riemann-Liouville fractional integral of order $\delta, f:[0, T] \times \mathbb{R} \rightarrow \mathbb{R}$ is a continuous function and $F:[0, T] \times \mathbb{R} \rightarrow \mathscr{P}(\mathbb{R})$ is a multivalued map $(\mathscr{P}(\mathbb{R})$ is the family of all nonempty subsets of $\mathbb{R}$ ).

The four-point boundary conditions in (1.7) can be interpreted as the value of the derivative of the unknown function at the nonlocal position $\xi$ is proportional to the value of the fractional derivative of the unknown function at the nonlocal position $\eta$, while the value of the unknown function at the right end point $(t=T)$ of the interval $[0, T]$ is proportional to the value of the fractional integral of the unknown function at the nonlocal position $\theta$.

The rest of this article is structured as follows. Some background material related to our work is presented in Section 2. In Section 3, existence and uniqueness results for problem (1.7) are obtained with the aid of the tools of the fixed point theory for single valued maps such as Sadovski fixed point theorem, Banach contraction mapping principle and Leray-Schauder nonlinear alternative, while the existence results for problem (1.8), based on the nonlinear alternative for contractive maps and CovitzNadler fixed point theorem, are proved in Section 4. Examples for illustration of our main results are presented. Finally, in Section 5, we discuss the boundary value problems for Riemann-Liouville type multi-term fractional differential equations and inclusions.

\section{PRELIMINARIES}

In this section, we introduce some notations and definitions of fractional calculus $[1,2]$ and present preliminary results needed in our proofs later.

Definition 2.1. The Riemann-Liouville fractional integral of order $\alpha>0$ for a function $g:(0, \infty) \rightarrow \mathbb{R}$ is defined by

$$
J^{\alpha} g(t)=\int_{0}^{t} \frac{(t-s)^{\alpha-1}}{\Gamma(\alpha)} g(s) d s
$$

provided that the right-hand side is point-wise defined on $(0, \infty)$, where $\Gamma$ is the Gamma function.

Definition 2.2. The Riemann-Liouville fractional derivative of order $\alpha>0$ of a continuous function $g:(0, \infty) \rightarrow \mathbb{R}$ is defined by

$$
D^{\alpha} g(t)=\frac{1}{\Gamma(n-\alpha)}\left(\frac{d}{d t}\right)^{n} \int_{0}^{t} \frac{g(s)}{(t-s)^{\alpha-n+1}} d s, \quad n-1<\alpha<n,
$$

where $n=[\alpha]+1,[\alpha]$ denotes the integer part of real number $\alpha$, provided that the right-hand side is point-wise defined on $(0, \infty)$. 
Definition 2.3. The Caputo derivative of order $q$ for a function $f:[0, \infty) \rightarrow \mathbb{R}$ can be written as

$$
{ }^{c} D^{q} f(t)=D^{q}\left(f(t)-\sum_{k=0}^{n-1} \frac{t^{k}}{k !} f^{(k)}(0)\right), \quad t>0, \quad n-1<q<n .
$$

Remark 2.4. If $f \in C^{n}[0, \infty)$, then

$$
{ }^{c} D^{q} f(t)=\frac{1}{\Gamma(n-q)} \int_{0}^{t} \frac{f^{(n)}(s)}{(t-s)^{q+1-n}} d s=I^{n-q} f^{(n)}(t), t>0, n-1<q<n .
$$

Lemma 2.5. If $\alpha+\beta>1$, then the equation $\left(I^{\alpha} I^{\beta} u\right)(t)=\left(I^{\alpha+\beta} u\right)(t), t \in J$ is satisfied for $u \in L^{1}(J, \mathbb{R})$.

Lemma 2.6. Let $\beta>\alpha$. Then the equation $\left(D^{\alpha} I^{\beta} u\right)(t)=\left(I^{\beta-\alpha} u\right)(t), t \in J$ is satisfied for $u \in C(J, \mathbb{R})$.

Lemma 2.7. Let $n=[\alpha]+1]$ if $\alpha \notin \mathbb{N}$ and $n=\alpha$ if $\alpha \in \mathbb{N}$. Then the following relations hold:

(i) for $k \in\{0,1,2, \ldots, n-1\}, D^{\alpha} t^{k}=0$;

(ii) if $\beta>n$ then $D^{\alpha} t^{\beta-1}=\frac{\Gamma(\beta)}{\Gamma(\beta-\alpha)} t^{\beta-\alpha-1}$;

(iii) $I^{\alpha} t^{\beta-1}=\frac{\Gamma(\beta)}{\Gamma(\beta+\alpha)} t^{\beta+\alpha-1}$.

Lemma 2.8. For $q>0$, the general solution of the fractional differential equation ${ }^{c} D^{q} x(t)=0$ is given by

$$
x(t)=c_{0}+c_{1} t+\ldots+c_{n-1} t^{n-1},
$$

where $c_{i} \in \mathbb{R}, i=0,1,2, \ldots, n-1(n=[q]+1)$.

In view of Lemma 2.8, it follows that

$$
I^{q c} D^{q} x(t)=x(t)+c_{0}+c_{1} t+\ldots+c_{n-1} t^{n-1},
$$

for some $c_{i} \in \mathbb{R}, i=0,1,2, \ldots, n-1(n=[q]+1)$.

Lemma 2.9. Let $y \in C([0, T], \mathbb{R})$ and

$$
\Omega=1-\frac{v \eta^{1-\gamma}}{\Gamma(2-\gamma)} \neq 0, \Lambda_{1}=1-\frac{\mu \theta^{\delta}}{\Gamma(1+\delta)} \neq 0, \Lambda_{2}=T-\frac{\mu \theta^{1+\delta}}{\Gamma(2+\delta)} .
$$

Then the unique solution of the linear boundary value problem:

$$
\left\{\begin{array}{l}
\lambda{ }^{C} D^{\alpha} x(t)+{ }^{C} D^{\beta} x(t)=y(t), t \in[0, T], \\
x^{\prime}(\xi)=v^{C} D^{\gamma} x(\eta), x(T)=\mu I^{\delta} x(\theta), \xi, \eta, \theta \in(0, T),
\end{array}\right.
$$

is given by

$$
\begin{aligned}
x(t)= & -\frac{1}{\lambda} I^{\alpha-\beta} x(t)+\frac{1}{\lambda} I^{\alpha} y(t) \\
& +\left(t-\frac{\Lambda_{2}}{\Lambda_{1}}\right) \frac{1}{\lambda \Omega}\left\{v\left(-I^{\alpha-\beta-\gamma} x(\eta)+I^{\alpha-\gamma} y(\eta)\right)+I^{\alpha-\beta-1} x(\xi)-I^{\alpha-1} y(\xi)\right\} \\
& +\frac{1}{\lambda \Lambda_{1}}\left\{\mu\left(-I^{\alpha-\beta+\delta} x(\theta)+I^{\alpha+\delta} y(\theta)\right)+I^{\alpha-\beta} x(T)-I^{\alpha} y(T)\right\} .
\end{aligned}
$$


Proof. Taking Riemann-Liouville fractional integral of order $\alpha$ of both sides of fractional differential equation in (2.3), we get

$$
x(t)=-\frac{1}{\lambda} I^{\alpha-\beta} x(t)+\frac{1}{\lambda} I^{\alpha} y(t)+c_{1} t+c_{2} .
$$

Applying the Caputo fractional derivative of order $\gamma$ to both sides of (2.5), we have

$$
{ }^{C} D^{\gamma} x(t)=-\frac{1}{\lambda} I^{\alpha-\beta-\gamma} x(t)+\frac{1}{\lambda} I^{\alpha-\gamma} y(t)+c_{1} \frac{t^{1-\gamma}}{\Gamma(2-\gamma)} .
$$

Taking the Riemann-Liouville integral of order $\delta$ of both sides of (2.5) yields

$$
I^{\delta} x(t)=-\frac{1}{\lambda} I^{\alpha-\beta+\delta} x(t)+\frac{1}{\lambda} I^{\alpha+\delta} y(t)+c_{1} \frac{t^{1+\delta}}{\Gamma(2+\delta)}+c_{2} \frac{t^{\delta}}{\Gamma(1+\delta)} .
$$

From (2.5), we have

$$
x^{\prime}(t)=-\frac{1}{\lambda} I^{\alpha-\beta-1} x(t)+\frac{1}{\lambda} I^{\alpha-1} y(t)+c_{1} .
$$

Using the above values in the boundary conditions of (2.3) and solving for $c_{1}$ and $c_{2}$, we get

$$
\begin{gathered}
c_{1}=\frac{1}{\Omega}\left\{-\frac{v}{\lambda} I^{\alpha-\beta-\gamma} x(\eta)+\frac{v}{\lambda} I^{\alpha-\gamma} y(\eta)+\frac{1}{\lambda} I^{\alpha-\beta-1} x(\xi)-\frac{1}{\lambda} I^{\alpha-1} y(\xi)\right\}, \\
c_{2}=-\frac{\Lambda_{2}}{\Lambda_{1}} c_{1}+\frac{1}{\Lambda_{1}}\left\{-\frac{\mu}{\lambda} I^{\alpha-\beta+\delta} x(\theta)+\frac{\mu}{\lambda} I^{\alpha+\delta} y(\theta)+\frac{1}{\lambda} I^{\alpha-\beta} x(T)-\frac{1}{\lambda} I^{\alpha} y(T)\right\} .
\end{gathered}
$$

Substituting the values of $c_{1}$ and $c_{2}$ into (2.5) completes solution (2.4). The converse follows by direct computation. The proof is completed.

For computational convenience, we set

$$
\begin{aligned}
Q_{1}= & \left(T+\frac{\left|\Lambda_{2}\right|}{\left|\Lambda_{1}\right|}\right) \frac{1}{|\lambda||\Omega|}\left\{\frac{|v| \eta^{\alpha-\beta-\gamma}}{\Gamma(\alpha-\beta-\gamma+1)}+\frac{\xi^{\alpha-\beta-1}}{\Gamma(\alpha-\beta)}\right\} \\
& +\frac{1}{|\lambda|\left|\Lambda_{1}\right|}\left\{\frac{|\mu| \theta^{\alpha-\beta+\delta}}{\Gamma(\alpha-\beta+\delta+1)}+\frac{T^{\alpha-\beta}}{\Gamma(\alpha-\beta+1)}\right\}+\frac{T^{\alpha-\beta}}{|\lambda| \Gamma(\alpha-\beta+1)}, \\
Q_{2}= & \left(T+\frac{\left|\Lambda_{2}\right|}{\left|\Lambda_{1}\right|}\right) \frac{1}{|\lambda||\Omega|}\left\{\frac{|v| \eta^{\alpha-\gamma}}{\Gamma(\alpha-\gamma+1)}+\frac{\xi^{\alpha-1}}{\Gamma(\alpha)}\right\} \\
& +\frac{1}{|\lambda|\left|\Lambda_{1}\right|}\left\{\frac{|\mu| \theta^{\alpha+\delta}}{\Gamma(\alpha+\delta+1)}+\frac{T^{\alpha}}{\Gamma(\alpha+1)}\right\}+\frac{T^{\alpha}}{|\lambda| \Gamma(\alpha+1)} .
\end{aligned}
$$

\section{EXISTENCE AND UNIQUENESS RESULTS FOR PROBLEM (1.7)}

Let $\mathscr{C}:=C(J, \mathbb{R})$ denote the Banach space of all continuous functions from $J$ into $\mathbb{R}$ with the norm $\|x\|=\sup \{|x(t)|, t \in J\}$.

3.1. Existence results via Sadovskii fixed point theorem. Our first existence result for problem (1.7) is based on Sadovskii fixed point theorem. Before proceeding further, let us recall some auxiliary material.

Definition 3.1. Let $M$ be a bounded set in metric space $(X, d)$. The Kuratowski measure of noncompactness, $\alpha(M)$, is defined as

$\inf \{\varepsilon: M$ covered by a finitely many sets such that the diameter of each set $\leq \varepsilon\}$. 
Definition 3.2. [22] Let $\Phi: \mathscr{D}(\Phi) \subseteq X \rightarrow X$ be a bounded and continuous operator on Banach space $X$. Then $\Phi$ is called a condensing map if $\alpha(\Phi(B))<\alpha(B)$ for all bounded sets $B \subset \mathscr{D}(\Phi)$, where $\alpha$ denotes the Kuratowski measure of noncompactness.

Lemma 3.3. [23, Example 11.7] The map $K+C$ is a $k$-set contraction with $0 \leq k<1$, and thus also condensing, if

(i) $K, C: \mathscr{D} \subseteq X \rightarrow X$ are operators on the Banach space $X$;

(ii) $K$ is $k$-contractive, that is, for all $x, y \in \mathscr{D}$ and a fixed $k \in[0,1)$,

$$
\|K x-K y\| \leq k\|x-y\|
$$

(iii) $C$ is compact.

Lemma 3.4. [24] Let $B$ be a convex, bounded and closed subset of a Banach space $X$ and $\Phi: B \rightarrow B$ be a condensing map. Then $\Phi$ has a fixed point.

Theorem 3.5. Let $f: J \times \mathbb{R} \rightarrow \mathbb{R}$ be a continuous function. Assume that:

$\left(H_{1}\right)$ there exists a function $p \in C\left(J, \mathbb{R}_{+}\right)$such that

$$
|f(t, u)| \leq p(t), \text { for a.e. } t \in J, \text { and each } u \in \mathbb{R}
$$

$\left(H_{2}\right) Q_{1}<1$, where $Q_{1}$ is defined by (2.8).

Then boundary value problem (1.7) has at least one solution on J.

Proof. Let $B_{r}=\{x \in \mathscr{C}:\|x\| \leq r\}$ be a closed bounded and convex subset of $\mathscr{C}$, where $r$ is a fixed constant. Consider the operator $\mathscr{A}: \mathscr{C} \rightarrow \mathscr{C}$ defined by

$$
\begin{aligned}
\mathscr{A} x(t)= & -\frac{1}{\lambda} I^{\alpha-\beta} x(t)+\frac{1}{\lambda} I^{\alpha} f(t, x(t)) \\
& +\left(t-\frac{\Lambda_{2}}{\Lambda_{1}}\right) \frac{1}{\lambda \Omega}\left\{v\left(-I^{\alpha-\beta-\gamma} x(\eta)+I^{\alpha-\gamma} f(\eta, x(\eta))\right)+I^{\alpha-\beta-1} x(\xi)-I^{\alpha-1} f(\xi, x(\xi))\right\} \\
& +\frac{1}{\lambda \Lambda_{1}}\left\{\mu\left(-I^{\alpha-\beta+\delta} x(\theta)+I^{\alpha+\delta} f(\theta, x(\theta))\right)+I^{\alpha-\beta} x(T)-I^{\alpha} f(T, x(T))\right\}, t \in J .
\end{aligned}
$$

Let us define $\mathscr{A}_{1}, \mathscr{A}_{2}: \mathscr{C} \rightarrow \mathscr{C}$ by

$$
\begin{aligned}
\mathscr{A}_{1} x(t)= & -\frac{1}{\lambda} I^{\alpha-\beta} x(t)+\left(t-\frac{\Lambda_{2}}{\Lambda_{1}}\right) \frac{1}{\lambda \Omega}\left\{-v I^{\alpha-\beta-\gamma} x(\eta)+I^{\alpha-\beta-1} x(\xi)\right\} \\
& +\frac{1}{\lambda \Lambda_{1}}\left\{-\mu I^{\alpha-\beta+\delta} x(\theta)+I^{\alpha-\beta} x(T)\right\}, t \in J
\end{aligned}
$$

and

$$
\begin{aligned}
\mathscr{A}_{2} x(t)= & \frac{1}{\lambda} I^{\alpha} f(t, x(t))+\left(t-\frac{\Lambda_{2}}{\Lambda_{1}}\right) \frac{1}{\lambda \Omega}\left\{v I^{\alpha-\gamma} f(\eta, x(\eta))-I^{\alpha-1} f(\xi, x(\xi))\right\} \\
& +\frac{1}{\lambda \Lambda_{1}}\left\{\mu I^{\alpha+\delta} f(\theta, x(\theta))-I^{\alpha} f(T, x(T))\right\}, t \in J .
\end{aligned}
$$

Clearly

$$
(\mathscr{A} x)(t)=\left(\mathscr{A}_{1} x\right)(t)+\left(\mathscr{A}_{2} x\right)(t), t \in J
$$

We shall show that $\mathscr{A}_{1}$ and $\mathscr{A}_{2}$ satisfy all the conditions of Lemma 3.4. The proof will be given in several steps. 
Step 1. $\mathscr{A} B_{r} \subset B_{r}$.

Let us select $r \geq \frac{\|p\| Q_{2}}{1-Q_{1}}$, where $Q_{1}$ and $Q_{2}$ are respectively given by (2.8) and (2.9). For any $x \in B_{r}$, we have

$$
\begin{aligned}
& \|\mathscr{A} x\| \\
\leq & \|x\| \frac{1}{|\lambda|} I^{\alpha-\beta}(1)(T)+\|p\| \frac{1}{|\lambda|} I^{\alpha}(1)(T)+\left(T+\frac{\left|\Lambda_{2}\right|}{\left|\Lambda_{1}\right|}\right) \frac{1}{|\Omega|}\left\{\|x\| \frac{|v|}{|\lambda|} I^{\alpha-\beta-\gamma}(1)(\eta)\right. \\
& \left.+\|p\| \frac{|v|}{|\lambda|} I^{\alpha-\gamma}(1)(\eta)+\|x\| \frac{1}{|\lambda|} I^{\alpha-\beta-1}(1)(\xi)+\|p\| \frac{1}{|\lambda|} I^{\alpha-1}(1)(\xi)\right\} \\
& +\frac{1}{\left|\Lambda_{1}\right|}\left\{\|x\| \frac{|\mu|}{|\lambda|} I^{\alpha-\beta+\delta}(1)(\theta)+\|p\| \frac{|\mu|}{|\lambda|} I^{\alpha+\delta}(1)(\theta)\right. \\
& \left.+\|x\| \frac{1}{\lambda} I^{\alpha-\beta}(1)(T)+\|p\| \frac{1}{|\lambda|} I^{\alpha}(1)(T)\right\} \\
\leq & \|x\|\left[\left(T+\frac{\left|\Lambda_{2}\right|}{\left|\Lambda_{1}\right|}\right) \frac{1}{|\lambda||\Omega|}\left\{\frac{|v| \eta^{\alpha-\beta-\gamma}}{\Gamma(\alpha-\beta-\gamma+1)}+\frac{\xi^{\alpha-\beta-1}}{\Gamma(\alpha-\beta)}\right\}\right. \\
& \left.+\frac{1}{|\lambda|\left|\Lambda_{1}\right|}\left\{\frac{|\mu| \theta^{\alpha-\beta+\delta}}{\Gamma(\alpha-\beta+\delta+1)}+\frac{T^{\alpha-\beta}}{\Gamma(\alpha-\beta+1)}\right\}+\frac{T^{\alpha-\beta}}{|\lambda| \Gamma(\alpha-\beta+1)}\right] \\
& +\|p\|\left[\left(T+\frac{\left|\Lambda_{2}\right|}{\left|\Lambda_{1}\right|}\right) \frac{1}{|\lambda||\Omega|}\left\{\frac{|v| \eta^{\alpha-\gamma}}{\Gamma(\alpha-\gamma+1)}+\frac{\xi^{\alpha-1}}{\Gamma(\alpha)}\right\}\right. \\
& \left.+\frac{1}{\left|\lambda \| \Lambda_{1}\right|}\left\{\frac{|\mu| \theta^{\alpha+\delta}}{\Gamma(\alpha+\delta+1)}+\frac{T^{\alpha}}{\Gamma(\alpha+1)}\right\}+\frac{T^{\alpha}}{|\lambda| \Gamma(\alpha+1)}\right] \\
\leq & r Q_{1}+\|p\| Q_{2} \leq r,
\end{aligned}
$$

which implies that $\mathscr{A} B_{r} \subset B_{r}$.

Step 2. $\mathscr{A}_{2}$ is compact.

Observe that the operator $\mathscr{A}_{2}$ is uniformly bounded in view of Step 1 . Let $t_{1}, t_{2} \in J$ with $t_{1}<t_{2}$ and $x \in B_{r}$. Then we have

$$
\begin{aligned}
& \left|\mathscr{A}_{2} x\left(t_{2}\right)-\mathscr{A}_{2} x\left(t_{1}\right)\right| \\
\leq & \frac{1}{|\lambda| \Gamma(\alpha)}\left[\int_{0}^{t_{1}}\left|\left(t_{2}-s\right)^{\alpha-1}-\left(t_{1}-s\right)^{\alpha-1}\right| p(s) d s+\int_{t_{1}}^{t_{2}}\left(t_{2}-s\right)^{\alpha-1} p(s) d s\right] \\
& +\frac{\left|t_{2}-t_{1}\right|}{|\lambda||\Omega|}\left\{\frac{v}{\Gamma(\alpha-\gamma)} \int_{0}^{\eta}(\eta-s)^{\alpha-\gamma-1} p(s) d s+\frac{1}{\Gamma(\alpha-1)} \int_{0}^{\xi}(\xi-s)^{\alpha-2} p(s) d s\right\} \\
\leq & \frac{\|p\|}{\Gamma(\alpha+1)}\left[2\left(t_{2}-t_{1}\right)^{\alpha}+\left|t_{2}^{\alpha}-t_{1}^{\alpha}\right|\right]+\frac{\|p\|\left|t_{2}-t_{1}\right|}{|\lambda||\Omega|}\left\{\frac{|v| \eta^{\alpha-\gamma}}{\Gamma(\alpha-\gamma+1)}+\frac{\xi^{\alpha-1}}{\Gamma(\alpha)}\right\},
\end{aligned}
$$

which is independent of $x$ and tends to zero as $t_{2}-t_{1} \rightarrow 0$. Thus, $\mathscr{A}_{2}$ is equicontinuous. Hence, by the Arzelá-Ascoli Theorem, $\mathscr{A}_{2}\left(B_{r}\right)$ is a relatively compact set.

Step 3. $\mathscr{A}_{1}$ is $Q_{1}$-contractive. 
Let $x, y \in B_{r}$. Then we have

$$
\begin{aligned}
& \left\|\mathscr{A}_{1} x-\mathscr{A}_{1} y\right\| \\
\leq & \frac{1}{|\lambda|} I^{\alpha-\beta}|x(t)-y(t)|+\left|t-\frac{\Lambda_{2}}{\Lambda_{1}}\right| \frac{1}{|\Omega|}\left\{\frac{|v|}{|\lambda|} I^{\alpha-\beta-\gamma}|x(\eta)-y(\eta)|\right. \\
& \left.+\frac{1}{|\lambda|} I^{\alpha-\beta-1}|x(\xi)-y(\xi)|\right\}+\frac{1}{\left|\Lambda_{1}\right|}\left\{\frac{|\mu|}{|\lambda|} I^{\alpha-\beta+\delta}|x(\theta)-y(\theta)|\right. \\
& \left.+\frac{1}{|\lambda|} I^{\alpha-\beta}|x(T)-y(T)|\right\} \\
\leq & {\left[\left(T+\frac{\left|\Lambda_{2}\right|}{\left|\Lambda_{1}\right|}\right) \frac{1}{|\lambda||\Omega|}\left\{\frac{|v| \eta^{\alpha-\beta-\gamma}}{\Gamma(\alpha-\beta-\gamma+1)}+\frac{\xi^{\alpha-\beta-1}}{\Gamma(\alpha-\beta)}\right\}\right.} \\
& +\frac{1}{|\lambda|\left|\Lambda_{1}\right|}\left\{\frac{|\mu| \theta^{\alpha-\beta+\delta}}{\Gamma(\alpha-\beta+\delta+1)}+\frac{T^{\alpha-\beta}}{\Gamma(\alpha-\beta+1)}\right\} \\
& \left.+\frac{T^{\alpha-\beta}}{|\lambda| \Gamma(\alpha-\beta+1)}\right]\|x-y\| \\
= & Q_{1}\|x-y\|,
\end{aligned}
$$

which is $Q_{1}$-contractive, since $Q_{1}<1$.

Step 4. $\mathscr{A}$ is condensing. Since $\mathscr{A}_{1}$ is continuous, $Q_{1}$-contraction and $\mathscr{A}_{2}$ is compact, therefore, by Lemma 3.3, $\mathscr{A}: B_{r} \rightarrow B_{r}$ with $\mathscr{A}=\mathscr{A}_{1}+\mathscr{A}_{2}$ is a condensing map on $B_{r}$.

From the above four steps, we conclude by Lemma 3.4 that $\mathscr{A}$ has a fixed point, which, in turn, implies that the problem (1.7) has a solution on $J$.

3.2. Uniqueness result via Banach fixed point theorem. In this section, we establish the uniqueness of solutions for the problem (1.7).

Theorem 3.6. Let $f:[0, T] \times \mathbb{R} \rightarrow \mathbb{R}$ be a continuous function. Assume that the following condition holds:

$\left(B_{1}\right)$ there exists $\ell>0$ such that

$$
|f(t, u)-f(t, v)| \leq \ell\|u-v\|, \quad \text { for } t \in J \text { and every } u, v \in \mathbb{R} .
$$

If

$$
Q_{1}+\ell Q_{2}<1
$$

where $Q_{1}, Q_{2}$ are defined by (2.8) and (2.9) respectively, then there exists a unique solution for the boundary value problem (1.7) on the interval $J$.

Proof. Consider the operator

$$
\mathscr{A}: C(J, \mathbb{R}) \rightarrow C(J, \mathbb{R})
$$

associated with problem (1.7) defined by (3.1). With $Q_{1}$ and $Q_{2}$ respectively given by (2.8) and (2.9), we fix

$$
r \geq \frac{Q_{2} f_{0}}{1-Q_{1}-\ell Q_{2}}, f_{0}=\sup _{t \in J}|f(t, 0)|,
$$


and show that $\mathscr{A} B_{r} \subset B_{r}$, where

$$
B_{r}=\{x \in C(J, \mathbb{R}):\|x\| \leq r\}
$$

For $x \in B_{r}$, using $\left(B_{1}\right)$, we get

$$
\begin{aligned}
& |\mathscr{A} x(t)| \\
& \leq \frac{1}{|\lambda|} I^{\alpha-\beta}|x(t)|+\frac{1}{|\lambda|} I^{\alpha}[|f(t, x(t))-f(t, 0)|+|f(t, 0)|] \\
& +\left(T+\frac{\left|\Lambda_{2}\right|}{\left|\Lambda_{1}\right|}\right) \frac{1}{|\Omega|}\left\{\frac{|v|}{|\lambda|} I^{\alpha-\beta-\gamma}|x(\eta)|+\frac{|v|}{|\lambda|} I^{\alpha-\gamma}[\mid f(\eta, x(\eta))\right. \\
& -f(\eta, 0)|+| f(\eta, 0) \mid]+\frac{1}{|\lambda|} I^{\alpha-\beta-1}|x(\xi)| \\
& \left.+\frac{1}{|\lambda|} I^{\alpha-1}[|f(\xi, x(\xi))-f(\xi, 0)|+|f(\xi, 0)|]\right\} \\
& +\frac{1}{\left|\Lambda_{1}\right|}\left\{\frac{|\mu|}{|\lambda|} I^{\alpha-\beta+\delta}|x(\theta)|+\frac{|\mu|}{|\lambda|} I^{\alpha+\delta}[|f(\theta, x(\theta))-f(\theta, 0)|+|f(\theta, 0)|]\right. \\
& \left.+\frac{1}{|\lambda|} I^{\alpha-\beta}|x(T)|+\frac{1}{|\lambda|} I^{\alpha}[|f(T, x(T))-f(T, 0)|+|f(T, 0)|]\right\} \\
& \leq\|x\| \frac{1}{|\lambda|} I^{\alpha-\beta}(1)(T)+\left(\ell\|x\|+f_{0}\right) \frac{1}{|\lambda|} I^{\alpha}(1)(T) \\
& +\left(T+\frac{\left|\Lambda_{2}\right|}{\left|\Lambda_{1}\right|}\right) \frac{1}{|\Omega|}\left\{\|x\| \frac{|v|}{|\lambda|} I^{\alpha-\beta-\gamma}(1)(\eta)\right. \\
& +\left(\ell\|x\|+f_{0}\right) \frac{|v|}{|\lambda|} I^{\alpha-\gamma}(1)(\eta)+\|x\| \frac{1}{|\lambda|} I^{\alpha-\beta-1}(1)(\xi) \\
& \left.+\left(\ell\|x\|+f_{0}\right) \frac{1}{|\lambda|} I^{\alpha-1}(1)(\xi)\right\} \\
& +\frac{1}{\left|\Lambda_{1}\right|}\left\{\|x\| \frac{|\mu|}{|\lambda|} I^{\alpha-\beta+\delta}(1)(\theta)+\left(\ell\|x\|+f_{0}\right) \frac{|\mu|}{|\lambda|} I^{\alpha+\delta}(1)(\theta)\right. \\
& \left.+\|x\| \frac{1}{|\lambda|} I^{\alpha-\beta}(1)(T)+\left(\ell\|x\|+f_{0}\right) \frac{1}{|\lambda|} I^{\alpha}(1)(T)\right\} \\
& \leq\|x\|\left[\left(T+\frac{\left|\Lambda_{2}\right|}{\left|\Lambda_{1}\right|}\right) \frac{1}{|\lambda||\Omega|}\left\{\frac{|v| \eta^{\alpha-\beta-\gamma}}{\Gamma(\alpha-\beta-\gamma+1)}+\frac{\xi^{\alpha-\beta-1}}{\Gamma(\alpha-\beta)}\right\}\right. \\
& \left.+\frac{1}{|\lambda|\left|\Lambda_{1}\right|}\left\{\frac{|\mu| \theta^{\alpha-\beta+\delta}}{\Gamma(\alpha-\beta+\delta+1)}+\frac{T^{\alpha-\beta}}{\Gamma(\alpha-\beta+1)}\right\}+\frac{T^{\alpha-\beta}}{|\lambda| \Gamma(\alpha-\beta+1)}\right] \\
& +\left(\ell\|x\|+f_{0}\right)\left[\left(T+\frac{\left|\Lambda_{2}\right|}{\left|\Lambda_{1}\right|}\right) \frac{1}{|\lambda||\Omega|}\left\{\frac{|v| \eta^{\alpha-\gamma}}{\Gamma(\alpha-\gamma+1)}+\frac{\xi^{\alpha-1}}{\Gamma(\alpha)}\right\}\right. \\
& \left.+\frac{1}{|\lambda|\left|\Lambda_{1}\right|}\left\{\frac{|\mu| \theta^{\alpha+\delta}}{\Gamma(\alpha+\delta+1)}+\frac{T^{\alpha}}{\Gamma(\alpha+1)}\right\}+\frac{T^{\alpha}}{|\lambda| \Gamma(\alpha+1)}\right] \\
& \leq r Q_{1}+\left(\ell r+f_{0}\right) Q_{2} \\
& \leq r \text {, }
\end{aligned}
$$

which, on taking the norm for $t \in J$, yields $\|\mathscr{A}(x)\| \leq r$. This shows that $\mathscr{A}$ maps $B_{r}$ into itself. 
In order to show that the operator $\mathscr{A}$ is a contraction, let $x, y \in C(J, \mathbb{R})$. Then, using (2.8) and (2.9), we get

$$
\begin{aligned}
& |\mathscr{A}(x)(t)-\mathscr{A}(y)(t)| \\
\leq & \frac{1}{|\lambda|} I^{\alpha-\beta}|x(t)-y(t)|+\frac{1}{|\lambda|} I^{\alpha}|f(t, x(t))-f(t, y(t))| \\
& +\left(T+\frac{\left|\Lambda_{2}\right|}{\left|\Lambda_{1}\right|}\right) \frac{1}{|\Omega|}\left\{\frac{|v|}{|\lambda|} I^{\alpha-\beta-\gamma}|x(\eta)-y(\eta)|+\frac{|v|}{|\lambda|} I^{\alpha-\gamma}|f(\eta, x(\eta))-| f(\eta, y(\eta)) \mid\right. \\
& \left.+\frac{1}{|\lambda|} I^{\alpha-\beta-1}|x(\xi)-y(\xi)|+\frac{1}{|\lambda|} I^{\alpha-1}|f(\xi, x(\xi))-f(\xi, y(\xi))|\right\} \\
& +\frac{1}{\left|\Lambda_{1}\right|}\left\{\frac{|\mu|}{|\lambda|} I^{\alpha-\beta+\delta}|x(\theta)-y(\theta)|+\frac{|\mu|}{|\lambda|} I^{\alpha+\delta}|f(\theta, x(\theta))-f(\theta, y(\theta))|\right. \\
& +\frac{1}{|\lambda|} I^{\alpha-\beta}|x(T)-y(T)|+\frac{1}{|\lambda|} I^{\alpha}[|f(T, x(T))-| f(T, y(T)) \mid\} \\
\leq & {\left[\left(T+\frac{\left|\Lambda_{2}\right|}{\left|\Lambda_{1}\right|}\right) \frac{1}{|\lambda||\Omega|}\left\{\frac{|v| \eta^{\alpha-\beta-\gamma}}{\Gamma(\alpha-\beta-\gamma+1)}+\frac{\xi^{\alpha-\beta-1}}{\Gamma(\alpha-\beta)}\right\}\right.} \\
& \left.+\frac{1}{|\lambda|\left|\Lambda_{1}\right|}\left\{\frac{|\mu| \theta^{\alpha-\beta+\delta}}{\Gamma(\alpha-\beta+\delta+1)}+\frac{T^{\alpha-\beta}}{\Gamma(\alpha-\beta+1)}\right\}+\frac{|\lambda| \Gamma(\alpha-\beta+1)}{|\alpha|}\right\}\|x-y\| \\
& +\left[\left(T+\frac{\left|\Lambda_{2}\right|}{\left|\Lambda_{1}\right|}\right) \frac{1}{|\lambda||\Omega|}\left\{\frac{|v| \eta^{\alpha-\gamma}}{\Gamma(\alpha-\gamma+1)}+\frac{\xi^{\alpha-1}}{\Gamma(\alpha)}\right\}\right. \\
& \left.+\frac{1}{|\lambda|\left|\Lambda_{1}\right|}\left\{\frac{|\mu| \theta^{\alpha+\delta}}{\Gamma(\alpha+\delta+1)}+\frac{T^{\alpha}}{\Gamma(\alpha+1)}\right\}+\frac{T^{\alpha}}{|\lambda| \Gamma(\alpha+1)}\right] \ell\|x-y\| \\
= & \left(Q_{1}+\ell Q_{2}\right)\|x-y\| .
\end{aligned}
$$

Consequently we obtain

$$
\|\mathscr{A}(x)-\mathscr{A}(y)\| \leq\left(Q_{1}+\ell Q_{2}\right)\|x-y\|,
$$

which, in view of (3.4), implies that $\mathscr{A}$ is a contraction. Hence $\mathscr{A}$ has a unique fixed point by Banach's contraction principle. This, in turn, shows that problem (1.7) has a unique solution on $J$. The proof is completed.

3.3. Existence results via Leray-Schauder nonlinear alternative. The next existence result is based on Leray-Schauder nonlinear alternative.

Lemma 3.7. (Nonlinear alternative for single valued maps) [22]. Let $E$ be a Banach space, $C$ a closed, convex subset of $E, U$ an open subset of $C$ and $0 \in U$. Suppose that $F: \bar{U} \rightarrow C$ is a continuous, compact (that is, $F(\bar{U})$ is a relatively compact subset of $C$ ) map. Then either

(i) F has a fixed point in $\bar{U}$, or

(ii) there is a $u \in \partial U$ (the boundary of $U$ in $C$ ) and $\lambda \in(0,1)$ with $u=\lambda F(u)$.

Theorem 3.8. Let $f:[0, T] \times \mathbb{R} \rightarrow \mathbb{R}$ be a continuous function satisfying $\left(H_{2}\right)$. Moreover, the following hypotheses hold:

$\left(C_{1}\right)$ there exist a continuous nondecreasing function $\psi:[0, \infty) \rightarrow(0, \infty)$ and a function $p \in C\left(J, \mathbb{R}^{+}\right)$ such that $|f(t, u)| \leq p(t) \psi(\|u\|)$ for each $(t, u) \in J \times \mathbb{R}$; 
$\left(C_{2}\right)$ there exists a constant $M>0$ such that

$$
\frac{\left(1-Q_{1}\right) M}{\|p\| \psi(M) Q_{2}}>1
$$

where $Q_{1}$ and $Q_{2}$ are defined by (2.8) and (2.9), respectively.

Then the boundary value problem (1.7) has at least one solution on $J$.

Proof. Consider the operator $\mathscr{A}: C(J, \mathbb{R}) \rightarrow C(J, \mathbb{R})$ defined by (3.1). We shall show that the operator $\mathscr{A}$ is continuous and completely continuous.

Step 1: $\mathscr{A}$ is continuous.

Let $\left\{x_{n}\right\}$ be a sequence such that $x_{n} \rightarrow x$ in $C(J, \mathbb{R})$. Then

$$
\begin{aligned}
& \left|\mathscr{A} x_{n}(t)-\mathscr{A} x(t)\right| \\
\leq & \frac{1}{|\lambda|} I^{\alpha-\beta}\left|x_{n}(t)-x(t)\right|+\frac{1}{|\lambda|} I^{\alpha}\left|f\left(t, x_{n}(t)\right)-f(t, x(t))\right| \\
& +\left(T+\frac{\left|\Lambda_{2}\right|}{\left|\Lambda_{1}\right|}\right) \frac{1}{|\Omega|}\left\{\frac{|v|}{|\lambda|} I^{\alpha-\beta-\gamma}\left|x_{n}(\eta)-x(\eta)\right|+\frac{|v|}{|\lambda|} I^{\alpha-\gamma}\left|f\left(\eta, x_{n}(\eta)\right)-\right| f(\eta, x(\eta)) \mid\right. \\
& \left.+\frac{1}{|\lambda|} I^{\alpha-\beta-1}\left|x_{n}(\xi)-x(\xi)\right|+\frac{1}{|\lambda|} I^{\alpha-1}\left|f\left(\xi, x_{n}(\xi)\right)-f(\xi, x(\xi))\right|\right\} \\
& +\frac{1}{\left|\Lambda_{1}\right|}\left\{\frac{|\mu|}{|\lambda|} I^{\alpha-\beta+\delta}\left|x_{n}(\theta)-x(\theta)\right|+\frac{|\mu|}{|\lambda|} I^{\alpha+\delta}\left|f\left(\theta, x_{n}(\theta)\right)-f(\theta, x(\theta))\right|\right. \\
& \left.+\frac{1}{|\lambda|} I^{\alpha-\beta}\left|x_{n}(T)-x(T)\right|+\frac{1}{|\lambda|} I^{\alpha}\left|f\left(T, x_{n}(T)\right)-f(T, x(T))\right|\right\} \\
\leq & Q_{1}\left\|x_{n}-x\right\|+Q_{2}\left\|f\left(\cdot, x_{n}\right)-f(\cdot, x)\right\| .
\end{aligned}
$$

Since $f$ is a continuous functions, therefore, we have

$$
\left\|\mathscr{A} x_{n}-\mathscr{A} x\right\| \leq Q_{1}\left\|x_{n}-x\right\|+Q_{2}\left\|f\left(\cdot, x_{n}\right)-f(\cdot, x)\right\| \rightarrow 0
$$

as $n \rightarrow \infty$.

Step 2: $\mathscr{A}$ maps bounded sets into bounded sets in $C(J, \mathbb{R})$.

Indeed, it is enough to show that, for any $r>0$, there exists a positive constant $\tilde{\ell}$ such that, for each $x \in B_{r}=\{x \in C(J, \mathbb{R}):\|x\| \leq r\}$, we have $\|\mathscr{A} x\| \leq \tilde{\ell}$. By $\left(C_{2}\right)$, for each $t \in J$, we have

$$
\begin{aligned}
|\mathscr{A} x(t)| \leq & \|x\| \frac{1}{|\lambda|} I^{\alpha-\beta}(1)(T)+\|p\| \psi(\|x\|) \frac{1}{|\lambda|} I^{\alpha}(1)(T) \\
& +\left(T+\frac{\left|\Lambda_{2}\right|}{\left|\Lambda_{1}\right|}\right) \frac{1}{|\Omega|}\left\{\|x\| \frac{|v|}{|\lambda|} I^{\alpha-\beta-\gamma}(1)(\eta)+\|p\| \psi(\|x\|) \frac{|v|}{|\lambda|} I^{\alpha-\gamma}(1)(\eta)\right. \\
& \left.+\|x\| \frac{1}{|\lambda|} I^{\alpha-\beta-1}(1)(\xi)+\|p\| \psi(\|x\|) \frac{1}{|\lambda|} I^{\alpha-1}(1)(\xi)\right\} \\
& +\frac{1}{\left|\Lambda_{1}\right|}\left\{\|x\| \frac{|\mu|}{|\lambda|} I^{\alpha-\beta+\delta}(1)(\theta)+\|p\| \psi(\|x\|) \frac{|\mu|}{|\lambda|} I^{\alpha+\delta}(1)(\theta)\right. \\
& \left.+\|x\| \frac{1}{|\lambda|} I^{\alpha-\beta}(1)(T)+\|p\| \psi(\|x\|) \frac{1}{|\lambda|} I^{\alpha}(1)(T)\right\}
\end{aligned}
$$




$$
\begin{aligned}
\leq & \|x\|\left[\left(T+\frac{\left|\Lambda_{2}\right|}{\left|\Lambda_{1}\right|}\right) \frac{1}{|\lambda||\Omega|}\left\{\frac{|v| \eta^{\alpha-\beta-\gamma}}{\Gamma(\alpha-\beta-\gamma+1)}+\frac{\xi^{\alpha-\beta-1}}{\Gamma(\alpha-\beta)}\right\}\right. \\
& \left.+\frac{1}{|\lambda|\left|\Lambda_{1}\right|}\left\{\frac{|\mu| \theta^{\alpha-\beta+\delta}}{\Gamma(\alpha-\beta+\delta+1)}+\frac{T^{\alpha-\beta}}{\Gamma(\alpha-\beta+1)}\right\}+\frac{T^{\alpha-\beta}}{|\lambda| \Gamma(\alpha-\beta+1)}\right] \\
& +\|p\| \psi(\|x\|)\left[\left(T+\frac{\left|\Lambda_{2}\right|}{\left|\Lambda_{1}\right|}\right) \frac{1}{|\lambda||\Omega|}\left\{\frac{|v| \eta^{\alpha-\gamma}}{\Gamma(\alpha-\gamma+1)}+\frac{\xi^{\alpha-1}}{\Gamma(\alpha)}\right\}\right. \\
& \left.+\frac{1}{|\lambda|\left|\Lambda_{1}\right|}\left\{\frac{|\mu| \theta^{\alpha+\delta}}{\Gamma(\alpha+\delta+1)}+\frac{T^{\alpha}}{\Gamma(\alpha+1)}\right\}+\frac{T^{\alpha}}{|\lambda| \Gamma(\alpha+1)}\right] \\
= & \|x\| Q_{1}+\|p\| \psi(\|x\|) Q_{2} .
\end{aligned}
$$

Thus

$$
\|\mathscr{A} x\| \leq r Q_{1}+\|p\| \psi(r) Q_{2}:=\tilde{\ell}
$$

Step 3: $\mathscr{A}$ maps bounded sets into equicontinuous sets of $C(J, \mathbb{R})$.

Let $t_{1}, t_{2} \in J, t_{1}<t_{2}, B_{r}$ be a bounded set of $C(J, \mathbb{R})$ as in Step 2, and let $x \in B_{r}$. Then

$$
\begin{aligned}
& \left|\mathscr{A}\left(x\left(t_{2}\right)\right)-\mathscr{A}\left(x\left(t_{1}\right)\right)\right| \\
\leq & \frac{1}{|\lambda| \Gamma(\alpha-\beta)}\left[\int_{0}^{t_{1}}\left|\left(t_{2}-s\right)^{\alpha-\beta-1}-\left(t_{1}-s\right)^{\alpha-\beta-1}\right||x(s)| d s+\int_{t_{1}}^{t_{2}}\left(t_{2}-s\right)^{\alpha-\beta-1}|x(s)| d s\right] \\
& +\frac{1}{|\lambda| \Gamma(\alpha)}\left[\int_{0}^{t_{1}}\left|\left(t_{2}-s\right)^{\alpha-1}-\left(t_{1}-s\right)^{\alpha-1}\right||f(s, x(s))| d s+\int_{t_{1}}^{t_{2}}\left(t_{2}-s\right)^{\alpha-1}|f(s, x(s))| d s\right] \\
& +\frac{\left|t_{2}-t_{1}\right|}{|\Omega|}\left\{\frac{|v|}{|\lambda| \Gamma(\alpha-\beta-\gamma)} \int_{0}^{\eta}(\eta-s)^{\alpha-\beta-\gamma-1}|x(s)| d s\right. \\
& +\frac{|v|}{|\lambda| \Gamma(\alpha-\gamma)} \int_{0}^{\eta}(\eta-s)^{\alpha-\gamma-1}|f(s, x(s))| d s+\frac{1}{|\lambda| \Gamma(\alpha-\beta-1)} \int_{0}^{\xi}(\xi-s)^{\alpha-\beta-2}|x(s)| d s \\
& \left.+\frac{1}{|\lambda| \Gamma(\alpha-1)} \int_{0}^{\xi}(\xi-s)^{\alpha-2}|f(s, x(s))| d s\right\} \\
\leq & \frac{r}{|\lambda| \Gamma(\alpha-\beta+1)}\left[2\left(t_{2}-t_{1}\right)^{\alpha-\beta}+\left|t_{2}^{\alpha-\beta}-t_{1}^{\alpha-\beta}\right|\right]+\frac{\|p\| \psi(r)}{|\lambda| \Gamma(\alpha+1)}\left[2\left(t_{2}-t_{1}\right)^{\alpha}+\left|t_{2}^{\alpha}-t_{1}^{\alpha}\right|\right] \\
& +\frac{\left|t_{2}-t_{1}\right|}{|\lambda||\Omega|}\left[r\left\{\frac{|v| \eta^{\alpha-\beta-\gamma}}{\Gamma(\alpha-\beta-\gamma+1)}+\frac{\xi^{\alpha-\beta-1}}{\Gamma(\alpha-\beta)}\right\}+\|p\| \psi(r)\left\{\frac{|v| \eta^{\alpha-\gamma}}{\Gamma(\alpha-\gamma+1)}+\frac{\xi^{\alpha-1}}{\Gamma(\alpha)}\right\}\right] .
\end{aligned}
$$

Clearly the right-hand side of the above inequality tends to zero independent of $x$ as $t_{1} \longrightarrow t_{2}$.

In consequence of Steps 1 to 3, it follows by the Arzelá-Ascoli theorem that the operator $\mathscr{A}: C(J, \mathbb{R}) \longrightarrow$ $C(J, \mathbb{R})$ is continuous and completely continuous.

Step 4: We show that there exists an open set $U \subseteq C(J, \mathbb{R})$ with $x \neq \sigma \mathscr{A}(x)$ for $\sigma \in(0,1)$ and $x \in \partial U$. 
Let $x \in C(J, \mathbb{R})$ and $x=\sigma \mathscr{A}(x)$ for some $0<\sigma<1$. Then, for each $t \in J$, we have

$$
\begin{aligned}
x(t)= & -\frac{\sigma}{\lambda} I^{\alpha-\beta} x(t)+\frac{\sigma}{\lambda} I^{\alpha} f(t, x(t))+\sigma\left(t-\frac{\Lambda_{2}}{\Lambda_{1}}\right) \frac{1}{\Omega}\left\{-\frac{v}{\lambda} I^{\alpha-\beta-\gamma} x(\eta)\right. \\
& \left.+\frac{v}{\lambda} I^{\alpha-\gamma} f(\eta, x(\eta))+\frac{1}{\lambda} I^{\alpha-\beta-1} x(\xi)-\frac{1}{\lambda} I^{\alpha-1} f(\xi, x(\xi))\right\} \\
& +\frac{\sigma}{\Lambda_{1}}\left\{-\frac{\mu}{\lambda} I^{\alpha-\beta+\delta} x(\theta)+\frac{\mu}{\lambda} I^{\alpha+\delta} f(\theta, x(\theta))+\frac{1}{\lambda} I^{\alpha-\beta} x(T)\right. \\
& \left.-\frac{1}{\lambda} I^{\alpha} f(T, x(T))\right\}, t \in J .
\end{aligned}
$$

As in Step 2, for each $t \in J$, it can be established that

$$
|x(t)| \leq\|x\| Q_{1}+\|p\| \psi(\|x\|) Q_{2},
$$

which can be expressed as

$$
\frac{\left(1-Q_{1}\right)\|x\|}{\|p\| \psi(\|x\|) Q_{2}} \leq 1
$$

In view of $\left(C_{2}\right)$, there exists $M$ such that $\|x\| \neq M$. Let us set

$$
U=\{x \in C(J, \mathbb{R}):\|x\|<M\} .
$$

Note that the operator $\mathscr{A}: \bar{U} \rightarrow C(J, \mathbb{R})$ is continuous and completely continuous. From the choice of $U$, there is no $x \in \partial U$ such that $x=\sigma \mathscr{A} x$ for some $\sigma \in(0,1)$. Consequently, by the nonlinear alternative of Leray-Schauder type (Lemma 3.7), we deduce that $\mathscr{A}$ has a fixed point $x \in \bar{U}$, which is a solution of the problem (1.7). This completes the proof.

Example 3.9. Let us consider the following boundary value problem

$$
\left\{\begin{array}{l}
9^{C} D^{8 / 5} x(t)+{ }^{C} D^{5 / 4} x(t)=f(t, x(t)), t \in J:=[0,2], \\
x^{\prime}(3 / 4)=(2 / 11){ }^{C} D^{1 / 5} x(1), x(2)=(1 / 7) I^{1 / 3} x(3 / 2),
\end{array}\right.
$$

where $\alpha=8 / 5, \beta=5 / 4, \gamma=1 / 5, \delta=1 / 3, \lambda=9, v=2 / 11, \mu=1 / 7, \xi=3 / 4, \eta=1, \theta=3 / 2, T=2$ and $f(t, x(t))$ will be fixed later.

Using the given data, we find that $|\Omega| \approx 0.8047870411, \Lambda_{1} \approx 0.8168708196, \Lambda_{2} \approx 1.793979672, Q_{1} \approx$ 0.7689763997 and $Q_{2} \approx 1.177019171$, where $\Omega, \Lambda_{1}, \Lambda_{2}, Q_{1}$, and $Q_{2}$ are given by (2.2), (2.8) and (2.9). For illustrating Theorem 3.5, we take

$$
f(t, x)=\frac{t e^{-t}}{30}\left(\frac{|x|}{|x|+1}+\cos x\right) .
$$

Clearly $f(t, x)$ is continuous and bounded by a positive real valued function $p(t)=\frac{t e^{-t}}{15}$. Also, the condition $\left(H_{2}\right)$ is satisfied, where $Q_{1}<1$. Hence all the conditions of Theorem 3.5 are satisfied and consequently the conclusion of Theorem 3.5 implies that the problem (3.6) with $f(t, x)$ given by (3.7) has at least one solution on $[0,2]$. Next, Theorem 3.6 can be illustrated by taking

$$
f(t, x)=\frac{\tan ^{-1} x+e^{-t}}{4 \sqrt{81+t}},
$$


where $f(t, x)$ is continuous and satisfies the condition $\left(B_{1}\right)$ with $\ell=1 / 36$. Also $Q_{1}+\ell Q_{2} \approx 0.8016713767$ $<1$. Thus, we conclude that there exist a unique solution on $[0,2]$ for the problem (3.6) with $f(t, x)$ given by (3.8). In order to illustrate Theorem 3.8, we choose

$$
f(t, x)=\frac{e^{-t} \cos t}{30}\left(\tan ^{-1} x+x+\frac{1}{8}\right) .
$$

It is easy to check that $f(t, x)$ is continuous and satisfy the condition $\left(C_{1}\right)$ with $p(t)=\frac{e^{-t} \cos t}{30}$ and $\psi=$ $\|x\|+\frac{4 \pi+1}{8}$. Also, by condition $C_{2}$, we find that $M>0.3469052365$. Thus, the hypothesis of Theorem 3.8 holds true, which implies that problem (3.6) with $f(t, x)$ given by (3.9) has at least one solution on $[0,2]$.

\section{EXISTENCE RESULTS FOR PROBLEM (1.8)}

First of all, we recall some basic concepts for multi-valued maps [25, 26, 27].

For a normed space $(X,\|\cdot\|)$, let $\mathscr{P}_{c l}(X)=\{Y \in \mathscr{P}(X): Y$ is closed $\}, \mathscr{P}_{b}(X)=\{Y \in \mathscr{P}(X)$ : $Y$ is bounded $\}, \mathscr{P}_{c p}(X)=\{Y \in \mathscr{P}(X): Y$ is compact $\}$ and $\mathscr{P}_{c p, c}(X)=\{Y \in \mathscr{P}(X): Y$ is compact and convex $\}$.

A multi-valued map $G: X \rightarrow \mathscr{P}(X):$

(i) is convex (closed) valued if $G(x)$ is convex (closed) for all $x \in X$;

(ii) is bounded on bounded sets if $G(\mathbb{B})=\cup_{x \in \mathbb{B}} G(x)$ is bounded in $X$ for all $\mathbb{B} \in \mathscr{P}_{b}(X)$ (i.e. $\left.\sup _{x \in \mathbb{B}}\{\sup \{|y|: y \in G(x)\}\}<\infty\right)$

(iii) is called upper semi-continuous (u.s.c.) on $X$ if for each $x_{0} \in X$, the set $G\left(x_{0}\right)$ is a nonempty closed subset of $X$, and if for each open set $N$ of $X$ containing $G\left(x_{0}\right)$, there exists an open neighborhood $\mathscr{N}_{0}$ of $x_{0}$ such that $G\left(\mathscr{N}_{0}\right) \subseteq N$;

(iv) $G$ is lower semi-continuous (l.s.c.) if the set $\{y \in X: G(y) \cap B \neq \emptyset\}$ is open for any open set $B$ in $E$;

(v) is said to be completely continuous if $G(\mathbb{B})$ is relatively compact for every $\mathbb{B} \in \mathscr{P}_{b}(X)$;

(vi) is said to be measurable if for every $y \in \mathbb{R}$, the function

$$
t \longmapsto d(y, G(t))=\inf \{|y-z|: z \in G(t)\}
$$

is measurable;

(vii) has a fixed point if there is $x \in X$ such that $x \in G(x)$. The fixed point set of the multivalued operator $G$ will be denoted by Fix $G$.

Definition 4.1. A multivalued map $F: J \times \mathbb{R} \rightarrow \mathscr{P}(\mathbb{R})$ is said to be Carathéodory if

(i) $t \longmapsto F(t, x)$ is measurable for each $x \in \mathbb{R}$;

(ii) $x \longmapsto F(t, x)$ is upper semicontinuous for almost all $t \in J$.

Further a Carathéodory function $F$ is called $L^{1}$-Carathéodory if

(iii) for each $\alpha>0$, there exists $\varphi_{\alpha} \in L^{1}\left(J, \mathbb{R}^{+}\right)$such that

$$
\|F(t, x)\|=\sup \{|v|: v \in F(t, x)\} \leq \varphi_{\alpha}(t)
$$

for all $x \in \mathbb{R}$ with $\|x\| \leq \alpha$ and for a.e. $t \in J$.

Recall that $\mathscr{C}:=C(J, \mathbb{R})$. For each $x \in \mathscr{C}$, define the set of selections of $F$ by

$$
S_{F, x}:=\left\{v \in L^{1}(J, \mathbb{R}): v(t) \in F(t, x(t)) \text { for a.e. } t \in J\right\} .
$$


We define the graph of $G$ to be the set $\operatorname{Gr}(G)=\{(x, y) \in X \times Y, y \in G(x)\}$ and recall two useful results regarding closed graphs and upper-semicontinuity.

Lemma 4.2. ([25, Proposition 1.2]) If $G: X \rightarrow \mathscr{P}_{c l}(Y)$ is u.s.c., then $G r(G)$ is a closed subset of $X \times Y$; i.e., for every sequence $\left\{x_{n}\right\}_{n \in \mathbb{N}} \subset X$ and $\left\{y_{n}\right\}_{n \in \mathbb{N}} \subset Y$, if when $n \rightarrow \infty, x_{n} \rightarrow x_{*}, y_{n} \rightarrow y_{*}$ and $y_{n} \in G\left(x_{n}\right)$, then $y_{*} \in G\left(x_{*}\right)$. Conversely, if $G$ is completely continuous and has a closed graph, then it is upper semi-continuous.

Lemma 4.3. ([28]) Let $X$ be a separable Banach space. Let $F: J \times \mathbb{R} \rightarrow \mathscr{P}_{c p, c}(X)$ be an $L^{1}-$ Carathéodory multivalued map and let $\Theta$ be a linear continuous mapping from $L^{1}(J, X)$ to $C(J, X)$. Then the operator

$$
\Theta \circ S_{F}: C(J, X) \rightarrow \mathscr{P}_{c p, c}(C(J, X)), x \mapsto\left(\Theta \circ S_{F}\right)(x)=\Theta\left(S_{F, x, y}\right)
$$

is a closed graph operator in $C(J, X) \times C(J, X)$.

4.1. The Carathéodory case. To prove our main result in this section, we use the following form of the nonlinear alternative for contractive maps [29, Corollary 3.8].

Theorem 4.4. Let $X$ be a Banach space, and $D$ a bounded neighborhood of $0 \in X$. Let $Z_{1}: X \rightarrow \mathscr{P}_{c p, c}(X)$ and $Z_{2}: \bar{D} \rightarrow \mathscr{P}_{c p, c}(X)$ two multi-valued operators satisfying

(a) $Z_{1}$ is contraction, and

(b) $Z_{2}$ is upper semi-continuous and compact.

Then, if $Q=Z_{1}+Z_{2}$, either

(i) $Q$ has a fixed point in $\bar{D}$ or

(ii) there is a point $u \in \partial D$ and $\lambda \in(0,1)$ with $u \in \lambda Q(u)$.

Definition 4.5. A function $x \in C^{1}(J, \mathbb{R})$ is a solution of the problem (1.8) if $x^{\prime}(\xi)=v^{C} D^{\gamma} x(\eta), x(T)=$ $\mu I^{\delta} x(\theta)$, and there exists function $v \in L^{1}(J, \mathbb{R})$ such that $v(t) \in F(t, x(t))$ a.e. on $J$ and

$$
\begin{aligned}
x(t)= & -\frac{1}{\lambda} I^{\alpha-\beta} x(t)+\frac{1}{\lambda} I^{\alpha} v(t)+\left(t-\frac{\Lambda_{2}}{\Lambda_{1}}\right) \frac{1}{\Omega}\left\{-\frac{v}{\lambda} I^{\alpha-\beta-\gamma} x(\eta)\right. \\
& \left.+\frac{v}{\lambda} I^{\alpha-\gamma} v(\eta)+\frac{1}{\lambda} I^{\alpha-\beta-1} x(\xi)-\frac{1}{\lambda} I^{\alpha-1} v(\xi)\right\} \\
& +\frac{1}{\Lambda_{1}}\left\{-\frac{\mu}{\lambda} I^{\alpha-\beta+\delta} x(\theta)+\frac{\mu}{\lambda} I^{\alpha+\delta} v(\theta)+\frac{1}{\lambda} I^{\alpha-\beta} x(T)-\frac{1}{\lambda} I^{\alpha} v(T)\right\},
\end{aligned}
$$

where $\Omega \neq 0, \Lambda_{1} \neq 0$ and $\Lambda_{2}$ are defined by (2.2).

Theorem 4.6. Let $f:[0, T] \times \mathbb{R} \rightarrow \mathbb{R}$ be a continuous function satisfying $\left(H_{2}\right)$. In addition, we assume that:

$\left(A_{1}\right) F: J \times \mathbb{R} \rightarrow \mathscr{P}_{c p, c}(\mathbb{R})$ is $L^{1}$-Carathéodory;

$\left(A_{2}\right)$ there exists a continuous nondecreasing function $\Phi:[0, \infty) \rightarrow(0, \infty)$ and a function $p \in C\left(J, \mathbb{R}^{+}\right)$ such that

$$
\|F(t, x)\|_{\mathscr{P}}:=\sup \{|y|: y \in F(t, x)\} \leq p(t) \Phi(\|x\|) \text { for each }(t, x) \in J \times \mathbb{R} ;
$$

$\left(A_{3}\right)$ there exists a constant $M>0$ such that

$$
\frac{\left(1-Q_{1}\right) M}{\|p\| \Phi(M) Q_{2}}>1
$$

where $Q_{1}$ and $Q_{2}$ are defined by (2.8) and (2.9) respectively. 
Then boundary value problem (1.8) has at least one solution on J.

Proof. To transform problem (1.8) into a fixed point problem, we define an operator $\mathscr{N}: \mathscr{C} \longrightarrow \mathscr{P}(\mathscr{C})$ by

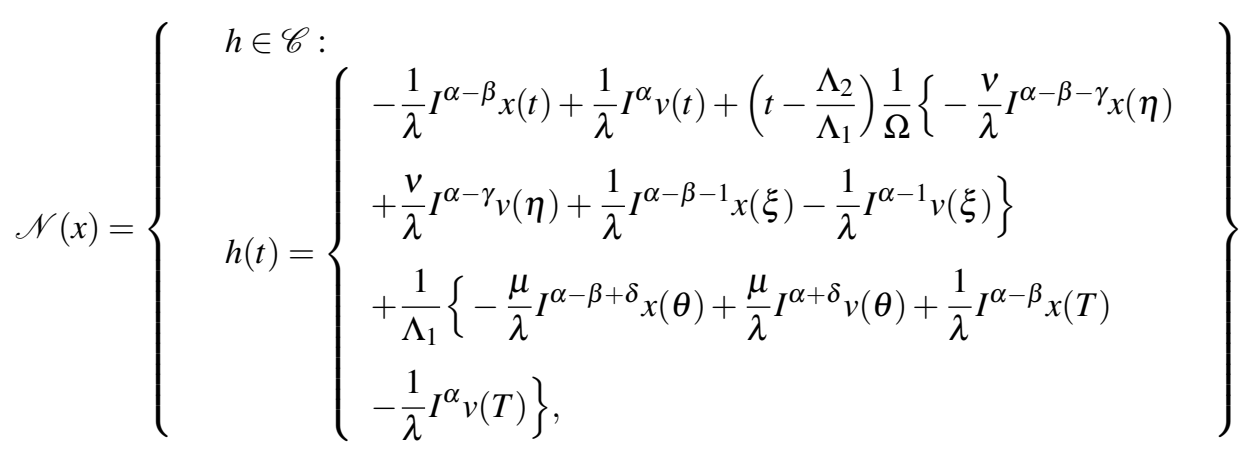

for $v \in S_{F, x}$.

Next we introduce the operator $\mathscr{B}_{1}: \mathscr{C} \longrightarrow \mathscr{C}$ by

$$
\begin{aligned}
\mathscr{B}_{1} x(t)= & -\frac{1}{\lambda} I^{\alpha-\beta} x(t)+\left(t-\frac{\Lambda_{2}}{\Lambda_{1}}\right) \frac{1}{\Omega}\left\{-\frac{v}{\lambda} I^{\alpha-\beta-\gamma} x(\eta)+\frac{1}{\lambda} I^{\alpha-\beta-1} x(\xi)\right\} \\
& +\frac{1}{\Lambda_{1}}\left\{-\frac{\mu}{\lambda} I^{\alpha-\beta+\delta} x(\theta)+\frac{1}{\lambda} I^{\alpha-\beta} x(T)\right\}, t \in J
\end{aligned}
$$

and the multi-valued operator $\mathscr{B}_{2}: \mathscr{C} \longrightarrow \mathscr{P}(\mathscr{C})$ by

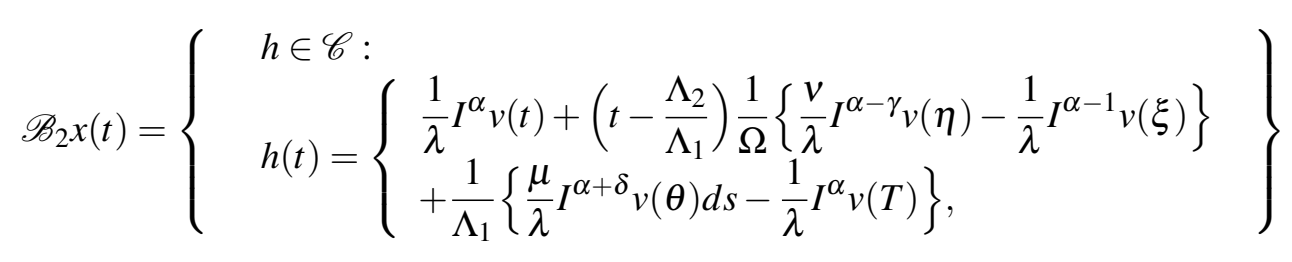

for $v \in S_{F, x}$. Observe that $\mathscr{N}=\mathscr{B}_{1}+\mathscr{B}_{2}$. We shall show that the operators $\mathscr{B}_{1}$ and $\mathscr{B}_{2}$ satisfy all the conditions of Theorem 4.4 on $J$. First, we show that the operators $\mathscr{B}_{1}$ and $\mathscr{B}_{2}$ define the multivalued operators $\mathscr{B}_{1}, \mathscr{B}_{2}: B_{r} \rightarrow \mathscr{P}_{c p, c}(\mathscr{C})$ where $B_{r}=\{x \in \mathscr{C}:\|x\| \leq r\}$ is a bounded set in $\mathscr{C}$. First we prove that $\mathscr{B}_{2}$ is compact-valued on $B_{r}$. Note that the operator $\mathscr{B}_{2}$ is equivalent to the composition $\mathscr{L} \circ S_{F}$, where $\mathscr{L}$ is the continuous linear operator on $L^{1}(J, \mathbb{R})$ into $\mathscr{C}$, defined by

$$
\begin{aligned}
\mathscr{L}(v)(t)= & \frac{1}{\lambda} I^{\alpha} v(t)+\left(t-\frac{\Lambda_{2}}{\Lambda_{1}}\right) \frac{1}{\Omega}\left\{\frac{v}{\lambda} I^{\alpha-\gamma} v(\eta)-\frac{1}{\lambda} I^{\alpha-1} v(\xi)\right\} \\
& +\frac{1}{\Lambda_{1}}\left\{\frac{\mu}{\lambda} I^{\alpha+\delta} v(\theta)-\frac{1}{\lambda} I^{\alpha} v(T)\right\} .
\end{aligned}
$$

Suppose that $x \in B_{r}$ is arbitrary and let $\left\{v_{n}\right\}$ be a sequence in $S_{F, x}$. Then, by definition of $S_{F, x}$, we have $v_{n}(t) \in F(t, x(t))$ for almost all $t \in J$. Since $F(t, x(t))$ is compact for all $t \in J$, there is a convergent subsequence of $\left\{v_{n}(t)\right\}$ (we denote it by $\left\{v_{n}(t)\right\}$ again) that converges in measure to some $v(t) \in S_{F, x}$ for almost all $t \in J$. On the other hand, $\mathscr{L}$ is continuous, so $\mathscr{L}\left(v_{n}\right)(t) \rightarrow \mathscr{L}(v)(t)$ pointwise on $J$. 
In order to show that the convergence is uniform, we have to show that $\left\{\mathscr{L}\left(v_{n}\right)\right\}$ is an equi-continuous sequence. Let $t_{1}, t_{2} \in J$ with $t_{1}<t_{2}$. Then, we have

$$
\begin{aligned}
& \left|\mathscr{L}\left(v_{n}\right)\left(t_{2}\right)-\mathscr{L}\left(v_{n}\right)\left(t_{1}\right)\right| \\
\leq & \frac{\Phi(r)}{|\lambda| \Gamma(\alpha)}\left[\int_{0}^{t_{1}}\left|\left(t_{2}-s\right)^{\alpha-1}-\left(t_{1}-s\right)^{\alpha-1}\right| p(s) d s+\int_{t_{1}}^{t_{2}}\left(t_{2}-s\right)^{\alpha-1} p(s) d s\right] \\
& +\frac{\Phi(r)\left|t_{2}-t_{1}\right|}{|\Omega|}\left\{\frac{|v|}{|\lambda| \Gamma(\alpha-\gamma)} \int_{0}^{\eta}(\eta-s)^{\alpha-\gamma-1} p(s) d s\right. \\
& \left.+\frac{1}{|\lambda| \Gamma(\alpha-1)} \int_{0}^{\xi}(\xi-s)^{\alpha-2} p(s) d s\right\} \\
\leq & \frac{\|p\| \Phi(r)}{\Gamma(\alpha+1)}\left[2\left(t_{2}-t_{1}\right)^{\alpha}+\left|t_{2}^{\alpha}-t_{1}^{\alpha}\right|\right]+\|p\| \Phi(r) \frac{\left|t_{2}-t_{1}\right|}{|\lambda||\Omega|}\left\{\frac{|v| \eta^{\alpha-\gamma}}{\Gamma(\alpha-\gamma+1)}+\frac{\xi^{\alpha-1}}{\Gamma(\alpha)}\right\} .
\end{aligned}
$$

We see that the right hand of the above inequality tends to zero as $t_{2} \rightarrow t_{1}$. Thus, the sequence $\left\{\mathscr{L}\left(v_{n}\right)\right\}$ is equi-continuous and by means the Arzelá-Ascoli theorem, we get that there is a uniformly convergent subsequence. So, there is a subsequence of $\left\{v_{n}\right\}$ (we denote it again by $\left\{v_{n}\right\}$ ) such that $\mathscr{L}\left(v_{n}\right) \rightarrow \mathscr{L}(v)$. Note that, $\mathscr{L}(v) \in \mathscr{L}\left(S_{F, x}\right)$. Hence, $\mathscr{B}_{2}(x)=\mathscr{L}\left(S_{F, x}\right)$ is compact for all $x \in B_{r}$. So $\mathscr{B}_{2}(x)$ is compact.

Now, we show that $\mathscr{B}_{2}(x)$ is convex for all $x \in \mathscr{C}$. Let $h_{1}, h_{2} \in \mathscr{B}_{2}(x)$. We select $v_{1}, v_{2} \in S_{F, x}$ such that

$$
\begin{aligned}
h_{i}(t)= & \frac{1}{\lambda} I^{\alpha} v_{i}(t)+\left(t-\frac{\Lambda_{2}}{\Lambda_{1}}\right) \frac{1}{\Omega}\left\{\frac{v}{\lambda} I^{\alpha-\gamma} v_{i}(\eta)-\frac{1}{\lambda} I^{\alpha-1} v_{i}(\xi)\right\} \\
& +\frac{1}{\Lambda_{1}}\left\{\frac{\mu}{\lambda} I^{\alpha+\delta} v_{i}(\theta)-\frac{1}{\lambda} I^{\alpha} v_{i}(T)\right\}, i=1,2,
\end{aligned}
$$

for almost all $t \in J$. Let $0 \leq \sigma \leq 1$. Then, we have

$$
\begin{aligned}
& {\left[\sigma h_{1}+(1-\sigma) h_{2}\right](t) } \\
= & \frac{1}{\lambda} I^{\alpha}\left[\sigma v_{1}(t)+(1-\sigma) v_{2}(t)\right]+\left(t-\frac{\Lambda_{2}}{\Lambda_{1}}\right) \frac{1}{\Omega}\left\{\frac{v}{\lambda} I^{\alpha-\gamma}\left[\sigma v_{1}(\eta)+(1-\sigma) v_{2}(\eta)\right]\right. \\
& \left.-\frac{1}{\lambda} I^{\alpha-1}\left[\sigma v_{1}(\xi)+(1-\sigma) v_{2}(\xi)\right]\right\}+\frac{1}{\Lambda_{1}}\left\{\frac{\mu}{\lambda} I^{\alpha+\delta}\left[\sigma v_{1}(\theta)+(1-\sigma) v_{2}(\theta)\right]\right. \\
& \left.-\frac{1}{\lambda} I^{\alpha}\left[\sigma v_{1}(T)+(1-\sigma) v_{2}(T)\right]\right\} .
\end{aligned}
$$

Since $F$ has convex values, we have that $S_{F, u}$ is convex and $\sigma v_{1}(s)+(1-\sigma) v_{2}(s) \in S_{F, x}$. Thus

$$
\sigma h_{1}+(1-\sigma) h_{2} \in \mathscr{B}_{2}(x) .
$$

Consequently, $\mathscr{B}_{2}$ is convex-valued. Obviously, $\mathscr{B}_{1}$ is compact and convex-valued.

The rest of the proof consists of several steps and claims.

Step 1: We show that $\mathscr{B}_{1}$ is a contraction on $\mathscr{C}$.

The proof is similar to that of Theorem 3.5 (Step 3) and we omit it.

Step 2: $\mathscr{B}_{2}$ is compact and upper semi-continuous. This will be established in several claims.

CLAIM I: $\mathscr{B}_{2}$ maps bounded sets into bounded sets in $\mathscr{C}$. Let $B_{r}=\{x \in \mathscr{C}:\|x\| \leq r\}$ be a bounded set in $\mathscr{C}$. Then, for each $h \in \mathscr{B}_{2}(x), x \in B_{r}$, there exists $v \in S_{F, x}$ such that

$$
h(t)=\frac{1}{\lambda} I^{\alpha} v(t)+\left(t-\frac{\Lambda_{2}}{\Lambda_{1}}\right) \frac{1}{\Omega}\left\{\frac{v}{\lambda} I^{\alpha-\gamma} v(\eta)-\frac{1}{\lambda} I^{\alpha-1} v(\xi)\right\}+\frac{1}{\Lambda_{1}}\left\{\frac{\mu}{\lambda} I^{\alpha+\delta} v(\theta)-\frac{1}{\lambda} I^{\alpha} v(T)\right\} .
$$


Then, for $t \in J$, we have

$$
\begin{aligned}
|h(t)| \leq & \|p\| \Phi(\|x\|)\left[\left(T+\frac{\left|\Lambda_{2}\right|}{\left|\Lambda_{1}\right|}\right) \frac{1}{|\lambda||\Omega|}\left\{\frac{|v| \eta^{\alpha-\gamma}}{\Gamma(\alpha-\gamma+1)}+\frac{\xi^{\alpha-1}}{\Gamma(\alpha)}\right\}\right. \\
& \left.+\frac{1}{|\lambda|\left|\Lambda_{1}\right|}\left\{\frac{|\mu| \theta^{\alpha+\delta}}{\Gamma(\alpha+\delta+1)}+\frac{T^{\alpha}}{\Gamma(\alpha+1)}\right\}+\frac{T^{\alpha}}{|\lambda| \Gamma(\alpha+1)}\right] .
\end{aligned}
$$

Thus,

$$
\begin{aligned}
\|h\| \leq & \|p\| \Phi(r)\left[\left(T+\frac{\left|\Lambda_{2}\right|}{\left|\Lambda_{1}\right|}\right) \frac{1}{|\lambda||\Omega|}\left\{\frac{|v| \eta^{\alpha-\gamma}}{\Gamma(\alpha-\gamma+1)}+\frac{\xi^{\alpha-1}}{\Gamma(\alpha)}\right\}\right. \\
& \left.+\frac{1}{|\lambda|\left|\Lambda_{1}\right|}\left\{\frac{|\mu| \theta^{\alpha+\delta}}{\Gamma(\alpha+\delta+1)}+\frac{T^{\alpha}}{\Gamma(\alpha+1)}\right\}+\frac{T^{\alpha}}{|\lambda| \Gamma(\alpha+1)}\right] .
\end{aligned}
$$

CLAIM II: $\mathscr{B}_{2}$ maps bounded sets into equi-continuous sets. The proof is based on the arguments employed in proving that $\left\{\mathscr{L}\left(v_{n}\right)\right\}$ is an equicontinuous sequence, so we do not repeat it here.

Next we show that $\mathscr{B}_{2}$ is an upper semi-continuous multi-valued mapping. It is known by Lemma 4.2 that $\mathscr{B}_{2}$ will be upper semicontinuous if we establish that it has a closed graph. Since $\mathscr{B}_{2}$ is already shown to be completely continuous, we only need to show that will prove that $\mathscr{B}_{2}$ has a closed graph.

ClAim III: $\mathscr{B}_{2}$ has a closed graph. Let $x_{n} \rightarrow x_{*}, h_{n} \in \mathscr{B}_{2}\left(x_{n}\right)$ and $h_{n} \rightarrow h_{*}$. Then we need to show that $h_{*} \in \mathscr{B}_{2}\left(x_{*}\right)$. Associated with $h_{n} \in \mathscr{B}_{2}\left(x_{n}\right)$, there exists $v_{n} \in S_{F, x_{n}}$ such that for each $t \in J$,

$$
\begin{aligned}
h_{n}(t)= & \frac{1}{\lambda} I^{\alpha} v_{n}(t)+\left(t-\frac{\Lambda_{2}}{\Lambda_{1}}\right) \frac{1}{\Omega}\left\{\frac{v}{\lambda} I^{\alpha-\gamma} v_{n}(\eta)-\frac{1}{\lambda} I^{\alpha-1} v_{n}(\xi)\right\} \\
& +\frac{1}{\Lambda_{1}}\left\{\frac{\mu}{\lambda} I^{\alpha+\delta} v_{n}(\theta)-\frac{1}{\lambda} I^{\alpha} v_{n}(T)\right\} .
\end{aligned}
$$

Thus it suffices to show that there exists $v_{*} \in S_{F, x_{*}}$ such that for each $t \in J$,

$$
\begin{aligned}
h_{*}(t)= & \frac{1}{\lambda} I^{\alpha} v_{*}(t)+\left(t-\frac{\Lambda_{2}}{\Lambda_{1}}\right) \frac{1}{\Omega}\left\{\frac{v}{\lambda} I^{\alpha-\gamma} v_{*}(\eta)-\frac{1}{\lambda} I^{\alpha-1} v_{*}(\xi)\right\} \\
& +\frac{1}{\Lambda_{1}}\left\{\frac{\mu}{\lambda} I^{\alpha+\delta} v_{*}(\theta)-\frac{1}{\lambda} I^{\alpha} v_{*}(T)\right\} .
\end{aligned}
$$

Let us consider the linear operator $\Theta: L^{1}(J, \mathbb{R}) \rightarrow \mathscr{C}$ given by

$$
\begin{aligned}
v \mapsto \Theta(v)(t)= & \frac{v}{\lambda} I^{\alpha} v(t)+\left(t-\frac{\Lambda_{2}}{\Lambda_{1}}\right) \frac{1}{\Omega}\left\{\frac{v}{\lambda} I^{\alpha-\gamma} v(\eta)-\frac{1}{\lambda} I^{\alpha-1} v(\xi)\right\} \\
& +\frac{1}{\Lambda_{1}}\left\{\frac{\mu}{\lambda} I^{\alpha+\delta} v(\theta)-\frac{1}{\lambda} I^{\alpha} v(t)\right\} .
\end{aligned}
$$

Observe that $\left\|h_{n}(t)-h_{*}(t)\right\| \rightarrow 0$, as $n \rightarrow \infty$. Thus, it follows by Lemma 4.3 that $\Theta \circ S_{F}$ is a closed graph operator. Further, we have $h_{n}(t) \in \Theta\left(S_{F, x_{n}}\right)$. Since $x_{n} \rightarrow x_{*}$, we have that

$$
\begin{aligned}
h_{*}(t)= & \frac{1}{\lambda} I^{\alpha} v_{*}(t)+\left(t-\frac{\Lambda_{2}}{\Lambda_{1}}\right) \frac{1}{\Omega}\left\{\frac{v}{\lambda} I^{\alpha-\gamma} v_{*}(\eta)-\frac{1}{\lambda} I^{\alpha-1} v_{*}(\xi)\right\} \\
& +\frac{1}{\Lambda_{1}}\left\{\frac{\mu}{\lambda} I^{\alpha+\delta} v_{*}(\theta)-\frac{1}{\lambda} I^{\alpha} v_{*}(T)\right\},
\end{aligned}
$$


for some $v_{*} \in S_{F, x_{*}}$. Hence $\mathscr{B}_{2}$ has a closed graph (and therefore has closed values). In consequence, the operator $\mathscr{B}_{2}$ is compact valued and upper semi-continuous.

Thus the operators $\mathscr{B}_{1}$ and $\mathscr{B}_{2}$ satisfy all the conditions of Theorem 4.4 and hence its conclusion implies either condition (i) or condition (ii) holds. We show that the conclusion (ii) is not possible. If $x \in \rho \mathscr{B}_{1}(x)+\rho \mathscr{B}_{2}(x)$ for $\rho \in(0,1)$, then there exist $v \in S_{F, x}$ such that

$$
\begin{aligned}
x(t)= & -\rho \frac{1}{\lambda} I^{\alpha-\beta} x(t)+\rho \frac{1}{\lambda} I^{\alpha} v(t)+\rho\left(t-\frac{\Lambda_{2}}{\Lambda_{1}}\right) \frac{1}{\Omega}\left\{-\frac{v}{\lambda} I^{\alpha-\beta-\gamma} x(\eta)\right. \\
& \left.+\frac{v}{\lambda} I^{\alpha-\gamma} v(\eta)+\frac{1}{\lambda} I^{\alpha-\beta-1} x(\xi)-\frac{1}{\lambda} I^{\alpha-1} v(\xi)\right\} \\
& +\rho \frac{1}{\Lambda_{1}}\left\{-\frac{\mu}{\lambda} I^{\alpha-\beta+\delta} x(\theta)+\frac{\mu}{\lambda} I^{\alpha+\delta} v(\theta)+\frac{1}{\lambda} I^{\alpha-\beta} x(T)-\frac{1}{\lambda} I^{\alpha} v(T)\right\} .
\end{aligned}
$$

By our assumptions, we have

$$
\begin{aligned}
|x(t)| \leq & \|x\|\left[\left(T+\frac{\left|\Lambda_{2}\right|}{\left|\Lambda_{1}\right|}\right) \frac{1}{|\lambda||\Omega|}\left\{\frac{|v| \eta^{\alpha-\beta-\gamma}}{\Gamma(\alpha-\beta-\gamma+1)}+\frac{\xi^{\alpha-\beta-1}}{\Gamma(\alpha-\beta)}\right\}\right. \\
& \left.+\frac{1}{|\lambda|\left|\Lambda_{1}\right|}\left\{\frac{|\mu| \theta^{\alpha-\beta+\delta}}{\Gamma(\alpha-\beta+\delta+1)}+\frac{T^{\alpha-\beta}}{\Gamma(\alpha-\beta+1)}\right\}+\frac{T^{\alpha-\beta}}{|\lambda| \Gamma(\alpha-\beta+1)}\right] \\
& +\|p\| \Phi(\|x\|)\left[\left(T+\frac{\left|\Lambda_{2}\right|}{\left|\Lambda_{1}\right|}\right) \frac{1}{|\lambda||\Omega|}\left\{\frac{|v| \eta^{\alpha-\gamma}}{\Gamma(\alpha-\gamma+1)}+\frac{\xi^{\alpha-1}}{\Gamma(\alpha)}\right\}\right. \\
& \left.+\frac{1}{\left|\lambda \| \Lambda_{1}\right|}\left\{\frac{|\mu| \theta^{\alpha+\delta}}{\Gamma(\alpha+\delta+1)}+\frac{T^{\alpha}}{\Gamma(\alpha+1)}\right\}+\frac{T^{\alpha}}{|\lambda| \Gamma(\alpha+1)}\right] \\
\leq & Q_{1}\|x\|+\|p\| \Phi(\|x\|) Q_{2} .
\end{aligned}
$$

Thus

$$
\left(1-Q_{1}\right)\|x\| \leq\|p\| \Phi(\|x\|) Q_{2} .
$$

If condition (ii) of Theorem 4.4 holds, then there exists $\rho \in(0,1)$ and $x \in \partial B_{M}\left(B_{M}=\{x \in C:\|x\|<\right.$ $M\}$ ) with $x=\rho \mathscr{N}(x)$. Then, $x$ is a solution of (1.8) with $\|x\|=M$. Now, by the inequality (4.3), we get

$$
\frac{\left(1-Q_{1}\right) M}{\|p\| \Phi(M) Q_{2}} \leq 1
$$

which contradicts (4.2). Hence, $\mathscr{N}$ has a fixed point on $J$ by Theorem 4.4, and consequently the problem (1.8) has a solution. This completes the proof.

4.2. The Lipschitz case. In this subsection we prove the existence of solutions for the problem (1.8) with a not necessary nonconvex valued right hand side, by applying a fixed point theorem for multivalued maps due to Covitz and Nadler [30].

Let $(X, d)$ be a metric space induced from the normed space $(X ;\|\cdot\|)$. Consider $H_{d}: \mathscr{P}(X) \times \mathscr{P}(X) \rightarrow$ $\mathbb{R} \cup\{\infty\}$ given by

$$
H_{d}(A, B)=\max \left\{\sup _{a \in A} d(a, B), \sup _{b \in B} d(A, b)\right\}
$$

where $d(A, b)=\inf _{a \in A} d(a ; b)$ and $d(a, B)=\inf _{b \in B} d(a ; b)$. Then $\left(\mathscr{P}_{c l, b}(X), H_{d}\right)$ is a metric space (see [31]).

Definition 4.7. A multivalued operator $N: X \rightarrow \mathscr{P}_{c l}(X)$ is called 
(a) $\gamma$-Lipschitz if and only if there exists $\gamma>0$ such that

$$
H_{d}(N(x), N(y)) \leq \gamma d(x, y) \text { for each } x, y \in X ;
$$

(b) a contraction if and only if it is $\gamma$-Lipschitz with $\gamma<1$.

Lemma 4.8. ([30]) Let $(X, d)$ be a complete metric space. If $N: X \rightarrow \mathscr{P}_{c l}(X)$ is a contraction, then FixN $\neq \emptyset$.

Theorem 4.9. Assume that:

$\left(D_{1}\right) F: J \times \mathbb{R} \rightarrow \mathscr{P}_{c p}(\mathbb{R})$ is such that $F(\cdot, x): J \rightarrow \mathscr{P}_{c p}(\mathbb{R})$ is measurable for each $x \in \mathbb{R} ;$

$\left(D_{2}\right) H_{d}(F(t, x), F(t, \bar{x})) \leq m(t)|x-\bar{x}|$ for almost all $t \in J$ and $x, \bar{x} \in \mathbb{R}$ with $m \in C\left(J, \mathbb{R}^{+}\right)$and $d(0, F(t, 0)) \leq$ $m(t)$ for almost all $t \in J$.

Then the boundary value problem (1.8) has at least one solution on $J$ if

$$
\varpi:=Q_{1}+\|m\| Q_{2}<1 .
$$

Proof. Consider the operator $\mathscr{N}$ defined at the begin of the proof of Theorem 4.6. Observe that the set $S_{F, x}$ is nonempty for each $x \in \mathscr{C}$ by the assumption $\left(D_{1}\right)$, so $\mathscr{N}$ has a measurable selection (see Theorem III.6 [32]). Now we show that the operator $\mathscr{N}$ satisfies the assumptions of Lemma 4.8. We show that $\mathscr{N}(x) \in \mathscr{P}_{c l}(\mathscr{C})$ for each $x \in \mathscr{C}$. Let $\left\{u_{n}\right\}_{n \geq 0} \in \mathscr{N}(x)$ be such that $u_{n} \rightarrow u(n \rightarrow \infty)$ in $\mathscr{C}$. Then $u \in \mathscr{C}$ and there exists $v_{n} \in S_{F, x_{n}}$ such that, for each $t \in J$,

$$
\begin{aligned}
u_{n}(t)= & -\frac{1}{\lambda} I^{\alpha-\beta} x_{n}(t)+\frac{1}{\lambda} I^{\alpha} v_{n}(t)+\left(t-\frac{\Lambda_{2}}{\Lambda_{1}}\right) \frac{1}{\Omega}\left\{-\frac{v}{\lambda} I^{\alpha-\beta-\gamma} x_{n}(\eta)\right. \\
& \left.+\frac{v}{\lambda} I^{\alpha-\gamma} v_{n}(\eta)+\frac{1}{\lambda} I^{\alpha-\beta-1} x_{n}(\xi)-\frac{1}{\lambda} I^{\alpha-1} v_{n}(\xi)\right\} \\
& +\frac{1}{\Lambda_{1}}\left\{-\frac{\mu}{\lambda} I^{\alpha-\beta+\delta} x_{n}(\theta)+\frac{\mu}{\lambda} I^{\alpha+\delta} v_{n}(\theta)+\frac{1}{\lambda} I^{\alpha-\beta} x_{n}(T)-\frac{1}{\lambda} I^{\alpha} v_{n}(T)\right\} .
\end{aligned}
$$

As $F$ has compact values, we pass onto a subsequence (if necessary) to obtain that $v_{n}$ converges to $v$ in $L^{1}(J, \mathbb{R})$. Thus, $v \in S_{F, x}$ and for each $t \in J$, we have

$$
\begin{aligned}
u_{n}(t) \rightarrow u(t)= & -\frac{1}{\lambda} I^{\alpha-\beta} x(t)+\frac{1}{\lambda} I^{\alpha} v(t)+\left(t-\frac{\Lambda_{2}}{\Lambda_{1}}\right) \frac{1}{\Omega}\left\{-\frac{v}{\lambda} I^{\alpha-\beta-\gamma} x(\eta)\right. \\
& \left.+\frac{v}{\lambda} I^{\alpha-\gamma} v(\eta)+\frac{1}{\lambda} I^{\alpha-\beta-1} x_{n}(\xi)-\frac{1}{\lambda} I^{\alpha-1} v(\xi)\right\} \\
& +\frac{1}{\Lambda_{1}}\left\{-\frac{\mu}{\lambda} I^{\alpha-\beta+\delta} x(\theta)+\frac{\mu}{\lambda} I^{\alpha+\delta} v(\theta)+\frac{1}{\lambda} I^{\alpha-\beta} x(T)-\frac{1}{\lambda} I^{\alpha} v(T)\right\},
\end{aligned}
$$

which implies that $u \in \mathscr{N}(x)$.

Next we show that there exists $\varpi<1$ (defined by (4.4)) such that

$$
H_{d}(\mathscr{N}(x), \mathscr{N}(\bar{x})) \leq \varpi\|x-\bar{x}\| \text { for each } x, \bar{x} \in C(J, \mathbb{R}) .
$$

Let $x, \bar{x} \in C(J, \mathbb{R})$ and $h_{1} \in \mathscr{N}(x)$. Then there exists $v_{1}(t) \in F(t, x(t))$ such that, for each $t \in J$,

$$
\begin{aligned}
h_{1}(t)= & -\frac{1}{\lambda} I^{\alpha-\beta} x(t)+\frac{1}{\lambda} I^{\alpha} v_{1}(t)+\left(t-\frac{\Lambda_{2}}{\Lambda_{1}}\right) \frac{1}{\Omega}\left\{-\frac{v}{\lambda} I^{\alpha-\beta-\gamma} x(\eta)\right. \\
& \left.+\frac{v}{\lambda} I^{\alpha-\gamma} v_{1}(\eta)+\frac{1}{\lambda} I^{\alpha-\beta-1} x(\xi)-\frac{1}{\lambda} I^{\alpha-1} v_{1}(\xi)\right\} \\
& +\frac{1}{\Lambda_{1}}\left\{-\frac{\mu}{\lambda} I^{\alpha-\beta+\delta} x(\theta)+\frac{\mu}{\lambda} I^{\alpha+\delta} v_{1}(\theta)+\frac{1}{\lambda} I^{\alpha-\beta} x(T)-\frac{1}{\lambda} I^{\alpha} v_{1}(T)\right\} .
\end{aligned}
$$


By $\left(D_{2}\right)$, we have

$$
H_{d}(F(t, x), F(t, \bar{x})) \leq m(t)|x(t)-\bar{x}(t)| .
$$

So, there exists $w \in F(t, \bar{x}(t))$ such that

$$
\left|v_{1}(t)-w\right| \leq m(t)|x(t)-\bar{x}(t)|, t \in J .
$$

Define $\widehat{U}: J \rightarrow \mathscr{P}(\mathbb{R})$ by

$$
\widehat{U}(t)=\left\{w \in \mathbb{R}:\left|v_{1}(t)-w\right| \leq m(t)|x(t)-\bar{x}(t)|\right\} .
$$

Since the multivalued operator $\widehat{U}(t) \cap F(t, \bar{x}(t))$ is measurable (Proposition III.4 [32]), there exists a function $v_{2}(t)$ which is a measurable selection for $\widehat{U}$. So $v_{2}(t) \in F(t, \bar{x}(t))$ and for each $t \in J$, we have

$$
\left|v_{1}(t)-v_{2}(t)\right| \leq m(t)|x(t)-\bar{x}(t)| .
$$

For each $t \in J$, let us define

$$
\begin{aligned}
h_{2}(t)= & -\frac{1}{\lambda} I^{\alpha-\beta} \bar{x}(t)+\frac{1}{\lambda} I^{\alpha} v_{2}(t)+\left(t-\frac{\Lambda_{2}}{\Lambda_{1}}\right) \frac{1}{\Omega}\left\{-\frac{v}{\lambda} I^{\alpha-\beta-\gamma} \bar{x}(\eta)\right. \\
& \left.+\frac{v}{\lambda} I^{\alpha-\gamma} v_{2}(\eta)+\frac{1}{\lambda} I^{\alpha-\beta-1} \bar{x}(\xi)-\frac{1}{\lambda} I^{\alpha-1} v_{2}(\xi)\right\} \\
& +\frac{1}{\Lambda_{1}}\left\{-\frac{\mu}{\lambda} I^{\alpha-\beta+\delta} \bar{x}(\theta)+\frac{\mu}{\lambda} I^{\alpha+\delta} v_{2}(\theta)+\frac{1}{\lambda} I^{\alpha-\beta} \bar{x}(T)-\frac{1}{\lambda} I^{\alpha} v_{2}(T)\right\} .
\end{aligned}
$$

Thus,

$$
\begin{aligned}
& \left|h_{1}(t)-h_{2}(t)\right| \\
\leq & \frac{1}{|\lambda|} I^{\alpha-\beta}|x(t)-\bar{x}(t)|+\frac{1}{|\lambda|} I^{\alpha}\left|v_{1}(t)-v_{2}(t)\right| \\
& +\left(T+\frac{\left|\Lambda_{2}\right|}{\left|\Lambda_{1}\right|}\right) \frac{1}{|\Omega|}\left\{\frac{|v|}{|\lambda|} I^{\alpha-\beta-\gamma}|x(\eta)-\bar{x}(\eta)|+\frac{|v|}{|\lambda|} I^{\alpha-\gamma}\left|v_{1}(\eta)-v_{2}(\eta)\right|\right. \\
& \left.+\frac{1}{|\lambda|} I^{\alpha-\beta-1}|x(\xi)-\bar{x}(\xi)|+\frac{1}{|\lambda|} I^{\alpha-1}\left|v_{1}(\xi)-v_{2}(\xi)\right|\right\} \\
& +\frac{1}{\left|\Lambda_{1}\right|}\left\{\frac{|\mu|}{|\lambda|} I^{\alpha-\beta+\delta}|x(\theta)-\bar{x}(\theta)|+\frac{|\mu|}{|\lambda|} I^{\alpha+\delta}\left|v_{1}(\theta)-v_{2}(\theta)\right|\right. \\
& \left.+\frac{1}{\lambda} I^{\alpha-\beta}|x(T)-\bar{x}(T)|+\frac{1}{|\lambda|} I^{\alpha}\left|v_{1}(T)-v_{2}(T)\right|\right\} \\
\leq & {\left[\left(T+\frac{\left|\Lambda_{2}\right|}{\left|\Lambda_{1}\right|}\right) \frac{1}{|\lambda||\Omega|}\left\{\frac{|v| \eta^{\alpha-\beta-\gamma}}{\Gamma(\alpha-\beta-\gamma+1)}+\frac{\xi^{\alpha-\beta-1}}{\Gamma(\alpha-\beta)}\right\}\right.} \\
& \left.+\frac{1}{|\lambda|\left|\Lambda_{1}\right|}\left\{\frac{|\mu| \theta^{\alpha-\beta+\delta}}{\Gamma(\alpha-\beta+\delta+1)}+\frac{T^{\alpha-\beta}}{\Gamma(\alpha-\beta+1)}\right\}+\frac{T^{\alpha-\beta}}{|\lambda| \Gamma(\alpha-\beta+1)}\right]\|x-\bar{x}\| \\
& +\left[\left(T+\frac{\left|\Lambda_{2}\right|}{\left|\Lambda_{1}\right|}\right) \frac{1}{|\lambda||\Omega|}\left\{\frac{|v| \eta^{\alpha-\gamma}}{\Gamma(\alpha-\gamma+1)}+\frac{\xi^{\alpha-1}}{\Gamma(\alpha)}\right\}\right. \\
& \left.+\frac{1}{|\lambda|\left|\Lambda_{1}\right|}\left\{\frac{|\mu| \theta^{\alpha+\delta}}{\Gamma(\alpha+\delta+1)}+\frac{T^{\alpha}}{\Gamma(\alpha+1)}\right\}+\frac{T^{\alpha}}{|\lambda| \Gamma(\alpha+1)}\right]\|m\|\|x-\bar{x}\| \\
= & \left(Q_{1}+\|m\| Q_{2}\right)\|x-\bar{x}\| .
\end{aligned}
$$


Hence,

$$
\left\|h_{1}-h_{2}\right\| \leq\left(Q_{1}+\|m\| Q_{2}\right)\|x-\bar{x}\| .
$$

Analogously, interchanging the roles of $x$ and $\bar{x}$, we obtain

$$
H_{d}(\mathscr{N}(x), \mathscr{N}(\bar{x})) \leq\left(Q_{1}+\|m\| Q_{2}\right)\|x-\bar{x}\| .
$$

So $\mathscr{N}$ is a contraction. Therefore, it follows by Lemma 4.8 that $\mathscr{N}$ has a fixed point $x$, which is a solution of (1.8). This completes the proof.

Example 4.10. Consider the following inclusions problem

$$
\left\{\begin{array}{l}
9^{C} D^{8 / 5} x(t)+{ }^{C} D^{5 / 4} x(t) \in F(t, x(t)), t \in J:=[0,2], \\
x^{\prime}(3 / 4)=(2 / 11){ }^{C} D^{1 / 5} x(1), x(2)=(1 / 7) I^{1 / 3} x(3 / 2),
\end{array}\right.
$$

where $F(t, x(t))$ will be fixed later.

In order to illustrate Theorem 4.6, we take

$$
F(t, x(t))=\left[\frac{e^{-t}}{2 \sqrt{900+t}}\left(\frac{|x|}{|x|+1}+x+\frac{1}{8}\right), \frac{(1+t)}{40}\left(\tan ^{-1} x+x+\frac{1}{3}\right)\right] .
$$

It is easy to check that $F(t, x(t))$ satisfies the conditions $\left(A_{1}\right)$ and $\left(A_{2}\right)$ with $p(t)=\frac{(1+t)}{40}$ and $\Phi(\|x\|)=$ $\|y\|+\frac{3 \pi+2}{6}$. Also by condition $\left(A_{3}\right)$, we have $M>1.177535025$. Thus, all the conditions of Theorem 4.6 hold true and consequently the problem (4.5) with $F(t, x)$ given by (4.6) has at least one solution on $[0,2]$.

Furthermore, Theorem 4.9 can be illustrated by choosing

$$
F(t, x(t))=\left[\frac{\cos t}{(30+t)}\left(\sin x+\frac{1}{2}\right), \frac{e^{-t}}{250}\left(\frac{|x|}{|x|+1}+t\right)\right] .
$$

Clearly

$$
H_{d}(F(t, x), F(t, \bar{x})) \leq \frac{1}{(30+t)}\|x-\bar{x}\| .
$$

Letting $m(t)=\frac{1}{30+t}$, it is easy to check that $d(0, F(t, 0)) \leq m(t)$ holds for almost all $t \in[0,2]$ and that $\varpi \approx 0.8082103721<1$ ( $\varpi$ is given by 4.4 ). As the hypotheses of Theorem 4.9 are satisfied, we conclude that the problem (4.5) with $F(t, x(t))$ given by (4.7) has at least one solution on [0,2].

\section{SiMILAR PROBLEMS}

Here we introduce boundary value problems involving Riemann-Liouville fractional derivatives instead of Caputo fractional derivative appeared in problem (1.7). Precisely, we consider the following boundary value problems

$$
\left\{\begin{array}{l}
\lambda{ }^{R L} D^{\alpha} x(t)+{ }^{R L} D^{\beta} x(t)=f(t, x(t)), t \in J:=[0, T] \\
x(0)=0, x(T)=\mu I^{\delta} x(\theta), \theta \in(0, T)
\end{array}\right.
$$

and

$$
\left\{\begin{array}{l}
\lambda^{R L} D^{\alpha} x(t)+{ }^{R L} D^{\beta} x(t)=f(t, x(t)), t \in J:=[0, T] \\
x(0)=0,{ }^{R L} D^{\gamma} x(\eta)=\mu I^{\delta} x(\theta), \eta, \theta \in(0, T)
\end{array}\right.
$$


where ${ }^{R L} D^{\chi}, \chi \in\{\alpha, \beta, \gamma\}$ is Riemann-Liouville fractional derivative of order $\chi$. We emphasize that for the case of Riemann-Liouville fractional derivative the condition $x(0)=0$ is necessary for the wellpossednes of the problem.

The solution for problem (5.1) is given by the integral equation

$$
\begin{aligned}
x(t)= & -\frac{1}{\lambda} I^{\alpha-\beta} x(t)+\frac{1}{\lambda} I^{\alpha} f(t, x(t))+t^{\alpha-1} \frac{1}{\Omega_{1}}\left\{-\frac{\mu}{\lambda} I^{\alpha-\beta+\delta} x(\theta)\right. \\
& \left.+\frac{\mu}{\lambda} I^{\alpha+\delta} f(\theta, x(\theta))+\frac{1}{\lambda} I^{\alpha+\beta} x(T)-\frac{1}{\lambda} I^{\alpha} f(T, x(T))\right\},
\end{aligned}
$$

where

$$
\Omega_{1}=T^{\alpha-1}-\frac{\mu \Gamma(\alpha)}{\Gamma(\alpha+\delta)} \theta^{\alpha+\delta-1} \neq 0,
$$

while the solution for problem (5.2) is

$$
\begin{aligned}
x(t)= & -\frac{1}{\lambda} I^{\alpha-\beta} x(t)+\frac{1}{\lambda} I^{\alpha} f(t, x(t))+t^{\alpha-1} \frac{1}{\Omega_{2}}\left\{-\frac{\mu}{\lambda} I^{\alpha-\beta+\delta} x(\theta)\right. \\
& \left.+\frac{\mu}{\lambda} I^{\alpha+\delta} f(\theta, x(\theta))+\frac{1}{\lambda} I^{\alpha-\beta-\gamma} x(\eta)-\frac{1}{\lambda} I^{\alpha-\gamma} f(\eta, x(\eta))\right\},
\end{aligned}
$$

where

$$
\Omega_{2}=\frac{\Gamma(\alpha)}{\Gamma(\alpha-\gamma)} \eta^{\alpha-\gamma-1}-\frac{\mu \Gamma(\alpha)}{\Gamma(\alpha+\delta)} \theta^{\alpha+\delta-1} \neq 0 .
$$

The existence results for the Riemann-Liouville boundary value problems (5.1) and (5.2) can be obtained by applying the strategy used in proving the results for Caputo type boundary value problem (1.7). To avoid the repetition, we omit the details. In a similar manner, one can deal with the the Riemann-Liouville inclusions problems associated with (5.1) and (5.2).

\section{CONCLUSIONS}

In this paper, We studied some new nonlocal fractional order boundary value problems. As a first problem, we considered a Caputo type multi-term fractional differential equation subject to nonlocal flux type and integral boundary conditions (problem (1.7)), and derived some existence and uniqueness results for this problem. It is imperative to note that Caputo type multi-term fractional differential equation (inclusions) for $\alpha=2$ and $\beta=3 / 2$ corresponds to a generalized nonlinear version of Bagley-Torvic equation (inclusions). Moreover, the results obtained for problem (1.7) specialize to some interesting new cases for appropriate choices of the parameters involved in it. For example, the results obtained for problem (1.7) are reduced to the ones for a nonlocal flux type terminal value problem when $\mu=0$. On the other hand, if we take $v=0$, our results become the ones for a nonlocal zero-flux and integroterminal value problem. For further enrichment of the literature on the topic, we considered the multivalued analogue of problem (1.7) and presented some existence results for it. We have also provided the outline for proving the existence of solutions for a Riemann-Liouville type multi-term fractional differential equation supplemented with $(i)$ Riemann-Liouville type integral boundary conditions and (ii) Riemann-Liouville type nonlocal integro-differential initial conditions. In a nutshell, the present work is innovative and interesting, and significantly contributes to the available materials on multi-term fractional differential equations and inclusions. 


\section{REFERENCES}

[1] I. Podlubny, Fractional Differential Equations, Academic Press, San Diego, 1999.

[2] A.A. Kilbas, H.M. Srivastava, J.J. Trujillo, Theory and Applications of Fractional Differential Equations, North-Holland Mathematics Studies, 204. Elsevier Science B.V., Amsterdam, 2006.

[3] J. Sabatier, O.P. Agrawal, J.A.T. Machado (Eds.), Advances in Fractional Calculus: Theoretical Developments and Applications in Physics and Engineering, Springer, Dordrecht, 2007.

[4] R.L. Bagley, P.J. Torvik, On the appearance of the fractional derivative in the behavior of real materials, J. Appl. Mech. 51 (1984), 294-298.

[5] B. Ahmad, S. K. Ntouyas, A. Alsaedi, New existence results for nonlinear fractional differential equations with three-point integral boundary conditions, Adv. Difference Equ. 2011 (2011), Article ID 10738.

[6] A. Alsaedi, S.K. Ntouyas, R.P. Agarwal, B. Ahmad, On Caputo type sequential fractional differential equations with nonlocal integral boundary conditions, Adv. Difference Equ. 2015 (2015), Article ID 33.

[7] B. Ahmad, S.K. Ntouyas, J. Tariboon, Fractional differential equations with nonlocal integral and integer-fractional-order Neumann type boundary conditions, Mediterr. J. Math. 13 (2016), 2365-2381.

[8] Z.B. Bai, W. Sun, Existence and multiplicity of positive solutions for singular fractional boundary value problems, Comput. Math. Appl. 63 (2012), 1369-1381.

[9] Y. Su, Z. Feng, Existence theory for an arbitrary order fractional differential equation with deviating argument, Acta Appl. Math. 118 (2012), 81-105.

[10] B. Ahmad, S.K. Ntouyas, Existence results for Caputo type sequential fractional differential inclusions with nonlocal integral boundary conditions, J. Appl. Math. Comput. 50 (2016), 157-174.

[11] A. Alsaedi, S.K. Ntouyas, B. Ahmad, New existence results for fractional integro-differential equations with nonlocal integral boundary conditions, Abstr. Appl. Anal. 2015 (2015), Article ID 205452.

[12] S.K. Ntouyas, S. Etemad, J. Tariboon, Existence of solutions for fractional differential inclusions with integral boundary conditions, Bound. Value Probl. 2015 (2015), Article ID 92.

[13] B. Ahmad, S.K. Ntouyas, Some fractional-order one-dimensional semi-linear problems under nonlocal integral boundary conditions, Rev. R. Acad. Cienc. Exactas Fysis. Nat. Ser. A-Mat. RACSAM, 110 (2016), 159-172.

[14] J.R. Graef, L. Kong, M. Wang, Existence and uniqueness of solutions for a fractional boundary value problem on a graph, Fract. Calc. Appl. Anal. 17 (2014), 499-510.

[15] J. Tariboon, S.K. Ntouyas, P. Thiramanus, Riemann-Liouville fractional differential equations with Hadamard fractional integral conditions, Inter. J. Appl. Math. Stat. 54 (2016), 119-134.

[16] B. Ahmad, R.P. Agarwal, Some new versions of fractional boundary value problems with slit-strips conditions, Bound. Value Probl. 2014 (2014), Article ID 175.

[17] J. Tariboon, S.K. Ntouyas, W. Sudsutad, Fractional integral problems for fractional differential equations via Caputo derivative, Adv. Difference Equ. 2014 (2014), Article ID 181.

[18] S.K. Ntouyas, J. Tariboon, Ch. Thaiprayoon, Nonlocal boundary value problems for Riemann-Liouville fractional differential inclusions with Hadamard fractional integral boundary conditions, Taiwanese J. Math. 20 (2016), 91-107.

[19] S. Niyom, S.K. Ntouyas, S. Laoprasittichok, J. Tariboon, Boundary value problems with four orders of Riemann-Liouville fractional derivatives, Adv. Difference Equ. 2016 (2016), Article ID 165.

[20] S.K. Ntouyas, J. Tariboon, Fractional boundary value problems with multiple orders of fractional derivatives and integrals, Electron. J. Differential Equations, 2017 (2017), Artile ID 100.

[21] L. Xu, Q. Dong, G. Li, Existence and Hyers-Ulam stability for three-point boundary value problems with RiemannLiouville fractional derivatives and integrals, Adv. Difference Equ. 2018 (2018), Article ID 458.

[22] A. Granas, J. Dugundji, Fixed Point Theory, Springer-Verlag, New York, 2005.

[23] E. Zeidler, Nonlinear functional analysis and its application: Fixed point-theorems, Springer-Verlag, New York, vol. 1 (1986).

[24] B.N. Sadovskii, On a fixed point principle, Funct. Anal. Appl. 1 (1967), 74-76.

[25] K. Deimling, Multivalued Differential Equations, Walter De Gruyter, Berlin-New York, 1992.

[26] Sh. Hu, N. Papageorgiou, Handbook of Multivalued Analysis, Volume I: Theory, Kluwer, Dordrecht, 1997.

[27] G.V. Smirnov, Introduction to the theory of differential inclusions, American Mathematical Society, Providence, RI, 2002. 
[28] A. Lasota, Z. Opial, An application of the Kakutani-Ky Fan theorem in the theory of ordinary differential equations, Bull. Acad. Polon. Sci. Ser. Sci. Math. Astronom. Phys. 13 (1965), 781-786.

[29] W.V. Petryshyn, P.M. Fitzpatric, A degree theory, fixed point theorems, and mapping theorems for multivalued noncompact maps, Trans. Amer. Math. Soc. 194 (1974), 1-25.

[30] H. Covitz, S. B. Nadler Jr., Multivalued contraction mappings in generalized metric spaces, Israel J. Math. 8 (1970), 5-11.

[31] M. Kisielewicz, Differential Inclusions and Optimal Control, Kluwer, Dordrecht, The Netherlands, 1991.

[32] C. Castaing, M. Valadier, Convex Analysis and Measurable Multifunctions, Lecture Notes in Mathematics 580, SpringerVerlag, Berlin-Heidelberg-New York, 1977. 